\title{
Effects of radiation on the metastatic process
}

\author{
Nora Sundahl $1^{1,2^{*}}$ D, Fréderic Duprez ${ }^{1}$, Piet Ost ${ }^{1,2}$, Wilfried De Neve ${ }^{1}$ and Marc Mareel ${ }^{1}$
}

\begin{abstract}
Radiotherapy remains one of the corner stones in the treatment of various malignancies and often leads to an improvement in overall survival. Nonetheless, pre-clinical evidence indicates that radiation can entail pro-metastatic effects via multiple pathways. Via direct actions on cancer cells and indirect actions on the tumor microenvironment, radiation has the potential to enhance epithelial-to-mesenchymal transition, invasion, migration, angiogenesis and metastasis. However, the data remains ambiguous and clinical observations that unequivocally prove these findings are lacking. In this review we discuss the pre-clinical and clinical data on the local and systemic effect of irradiation on the metastatic process with an emphasis on the molecular pathways involved.
\end{abstract}

Keywords: Radiation, Radiotherapy, Invasion, Metastasis, Cancer

\section{Background}

Local invasion and distant metastasis are the cause of death in most malignant tumors. Tumor response, reflected by improved overall survival, and toxic side effects, deteriorating quality of life, receive major attention in clinical trials investigating the main treatment modalities used in cancer, namely, surgery, radiotherapy, chemotherapy, targeted therapy and immunotherapy. Yet, far less attention is paid to the effects of these treatments on tumor progression as reflected by the metastatic process.

Malignant tumors consist of cancer cells and tumorassociated host cells, both participating in invasion and distant metastasis. These cells form ecosystems at the primary and at the metastatic site, mutually communicating with one another and with stem cell-generating organs such as the bone marrow. It is highly probable that therapeutic manipulation of one ecosystem affects the others, a phenomenon that needs to be analyzed in view of the increasing cellular and molecular complexity of therapy responses (Barker et al., 2015).

Metastatic cancer cells are released from the primary tumor or from other metastases, at an undefined moment of its development, to arrive in the circulation and

\footnotetext{
* Correspondence: nora.sundahl@ugent.be

'Department of Radiation-Oncology, Ghent University Hospital, C. Heymanslaan 10, 9000 Ghent, Belgium

${ }^{2}$ Immuno-Oncology Network Ghent (ION Ghent), Ghent, Belgium
}

home at distant sites, where the ecosystem permits them to survive and either remain dormant as micrometastases or grow to form macro-metastases (Mareel et al., 2009a). There is good evidence that cancer cells disseminate from the primary site early during tumor development (Hosseini et al., 2016), yet it is difficult to predict whether disseminated cancer cells are present at the moment of treatment and, if so, where they reside. Such cells are described as disseminated tumor cells (DTC) (Sosa et al., 2014) or sometimes as circulating tumor cells (CTC) (Kim et al., 2009). They can be awakened from dormancy by local therapeutic manipulation generating unfavorable distant effects.

Here we review the preclinical evidence on the effect of irradiation on three main steps in the metastatic process as proposed by Talmadge et al. (Talmadge \& Fidler, 2010), namely angiogenesis, motility and invasion, and metastasis with an emphasis on the molecular pathways involved. Subsequently, the clinical evidence on this subject is reviewed.

\section{Main text \\ Preclinical evidence \\ Angiogenesis}

One of the first molecules implicated in enhancement of distant metastasis after irradiation of the primary tumor was angiostatin, produced by the primary tumor and keeping metastasis dormant. Elimination of the primary 
tumor, either by irradiation or by surgery, shifts the balance towards pro-angiogenesis and growth of the lung metastases (Table 1) (Camphausen et al., 2001). Molecular communication between ecosystems is also witnessed by the vasculogenic and pro-metastatic tumor bed effect as discussed by Kuonen et al. (Kuonen et al., 2012d)

Table 1 In vitro and in vivo experiments investigating the influence of irradiation on angiogenesis

\begin{tabular}{|c|c|c|c|c|c|c|c|c|}
\hline \multicolumn{3}{|c|}{ Radiation } & \multirow[t]{2}{*}{ Vessel origin } & \multirow[t]{2}{*}{ Host } & \multicolumn{2}{|l|}{ Result } & \multirow[t]{2}{*}{ Molecular } & \multirow[t]{2}{*}{ Ref } \\
\hline Target & Mode & Dose & & & Type & IR/Ctr| & & \\
\hline \multicolumn{9}{|c|}{ Endothelial cells } \\
\hline & \multirow[t]{2}{*}{ C-ion } & \multirow[t]{2}{*}{$0.1-8 \mathrm{~Gy}$} & \multirow[t]{2}{*}{ HUVEC } & Transwell chamber & Migration & $<1$ & \multirow[t]{4}{*}{ avß3; MMP-2 } & \multirow[t]{4}{*}{ (Takahashi et al., 2003) } \\
\hline & & & & Collagen & Tube formation & $<1$ & & \\
\hline & \multirow[t]{2}{*}{$\mathrm{kV}$} & \multirow[t]{2}{*}{$0.1-8$ Gy } & & \multicolumn{2}{|l|}{ Transwell chamber } & $>1$ & & \\
\hline & & & & Collagen & Tube formation & 1 & & \\
\hline & $\mathrm{nm}$ & 4 Gy & HUVEC & Matrigel & Tube formation & 0.8 & \multirow[t]{2}{*}{$\operatorname{av\beta 3}$} & (Abdollahi et al., 2005) \\
\hline & $\mathrm{nm}$ & 8 Gy & HMEC & Matrigel & Invasion & 1 & & (Kaliski et al., 2005) \\
\hline & \multirow[t]{2}{*}{$250 \mathrm{kV}$} & \multirow[t]{2}{*}{6 Gy } & HUVEC & Tissue culture plastic & Wound healing migration & 4 & \multirow[t]{2}{*}{ eNOS } & \multirow[t]{2}{*}{ (Sonveaux et al., 2003) } \\
\hline & & & HUVEC & Matrigel & Tube formation & & & \\
\hline & ${ }^{137} \mathrm{Cs}$ & 3 Gy & HUVEC & Matrigel & Tube formation & 0.8 & $\operatorname{av} \beta 3$ & (Albert et al., 2006) \\
\hline & $125 \mathrm{kV}$ & 15 Gy & HUVEC & Tissue culture plastic & Wound healing migration & 0.6 & & (Imaizumi et al., 2010) \\
\hline & $6 \mathrm{MV}$ & 0.5 Gy & HUVEC & & Wound healing migration & $>1$ & VEGFR & (Sofia Vala et al., 2010) \\
\hline \multicolumn{9}{|c|}{ Cancer cells } \\
\hline & $6 \mathrm{MV}$ & $10 \mathrm{~Gy}$ & HUVEC & Transwell/CM C6 & Chemotactic migration & 2 & MMP-2 \& -9 & (Parthymou et al., 2004) \\
\hline & \multirow[t]{3}{*}{$6 \mathrm{MV}$} & \multirow[t]{2}{*}{$10 \mathrm{~Gy}$} & CAM & CAM/C6 & angiogenesis & $>1$ & & (Parthymou et al., 2004) \\
\hline & & & HUVEC & Matrigel/PC3 & Invasion & 1.9 & & (Abdollahi et al., 2005) \\
\hline & & 8 Gy & HMEC & Matrigel/CM B16 & Invasion & 2.4 & MMP-2 & (Kaliski et al., 2005) \\
\hline & 150 kV & $10 \mathrm{~Gy}$ & HMEC & Matrigel/CM IOMM & Tube formation & 1.4 & UPA & (Kargiotis et al., 2008) \\
\hline & $\mathrm{nm}$ & 8 Gy & HMEC & T. c. plastic/CM U251 & Branching & 1.4 & MMP-2 & (Badiga et al., 2011) \\
\hline \multicolumn{9}{|l|}{ CAF } \\
\hline & \multirow[t]{2}{*}{$15 \mathrm{MV}$} & \multirow[t]{2}{*}{$18 \mathrm{~Gy}$} & HUVEC & Transwell/CM CAF & Migration & 0.6 & & (Hellevik et al., 2013) \\
\hline & & & HUVEC & Matrigel/CM CAF & Tube formation & 1 & & (Hellevik et al., 2013) \\
\hline Inoculati & site & & & & & & & \\
\hline & $50 \mathrm{kV}$ & 19 Gy & CAM & CAM/C6 & Angiogenesis & $>1(48 \mathrm{~h})$ & & (Polytarchou et al., 2004) \\
\hline & $6 \mathrm{MV}$ & 2 Gy & SCID mouse subcutis & HepG2 & Micro-vessel density & $>1$ & VEGF & \\
\hline & 125 kV & 20 Gy & C57/BI subcutis & Matrigel plus FGF2 & Micro-vessel density & 0.36 & FGF2 & (Imaizumi et al., 2010) \\
\hline & & & & Matrigel plus VEGF & Micro-vessel density & 0.11 & VEGF & \\
\hline & $6 \mathrm{MV}$ & $0.5 \mathrm{~Gy}$ & nude mouse subcutis & Matrigel plus FGF2 & Angiogenesis & $>1$ & & (Sofia Vala et al., 2010) \\
\hline & 125 kV & 20 Gy & mammary fat pad. & Mammary/AT1 & Micro-vessel density & 0.44 & & (Kuonen et al., 2012b) \\
\hline Total bo & & & & & & & & \\
\hline & 220 kV & 15 Gy & C57/Bl aortic & Tissue culture plastic & Sprouting & 0.11 & TGFI $\beta$ & (Imaizumi et al., 2010) \\
\hline & $6 \mathrm{MV}$ & 0.5 Gy & zebra fish & Embryo & Angiogenesis & $>1$ & & (Sofia Vala et al., 2010) \\
\hline Tumor & & & & & & & & \\
\hline & $250 \mathrm{kV}$ & 6 Gy & C57/Bl6 subcutis & LLC & Micro-vessel density & $>1$ & & (Sonveaux et al., 2003) \\
\hline & $6 \mathrm{MV}$ & 8 Gy & C57/BL6 cerebrum & ALTS1C1 & Micro-vessel density & $>1$ & & (Wang et al., 2013) \\
\hline & & & SCID mouse subcutis & SW410 & Micro-vessel density & 2 & & (Timaner et al., 2015) \\
\hline
\end{tabular}

Abbreviations: avb3, integrin; ALTS1C1, SV40 large T-transformed astrocytes; B16, mouse melanoma cells; C57/BL, mouse strain; C6, rat glioma cells; CAF, cancer-associated fibroblasts; CAM, chick chorioallantoic membrane; C-ion, carbon ion; CM, conditioned medium; eNOS, endothelial nitric oxide synthase; FGF2, fibroblast growth factor 2; HepG2, human hepatoma cells; HMEC, human microvascular endothelial cells; HUVEC, human umbilical vein endothelial cells; IR/Ctrl, irradiated over unirradiated control target; IOMM, human meningioma cells; LLC, Lewis lung cancer cells; MMP, matrix metalloproteinase; MVD, microvessel density; nm, not mentioned; PC3, human prostate cancer cells; pcb, polycarbonate transwell chamber; SW410, human colon carcinoma cells; t.c., tissue culture; TGFb, transforming growth factor beta; U252, human glioma cells; uPA, urokinase-type plasminogen activator; VEGF, vascular endothelial growth factor; VEGFR, vascular endothelial growth factor receptor; WH, wound healing 
Irradiation-induced suppression of angiogenesis creates a hypoxic primary tumor ecosystem. Hypoxia stimulates hypoxia inducible factor (HIF)-dependent expression of CXCL12 and KITL promoting mobilization from the bone marrow and recruitment to primary tumor and metastatic sites of $\mathrm{CXCR}_{4}^{+} \mathrm{CD} 11 \mathrm{~b}^{+}$bone marrow-derived cells and

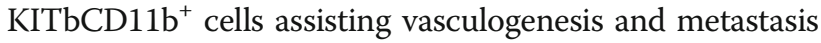
respectively (Kuonen et al., 2012d). Recruitment of

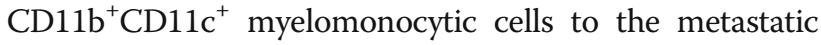
site was also found after whole thorax irradiation at a dose of 15 Gy of mice that significantly enhanced seeding and metastatic growth of intravenously injected cancer cells. Such treatment was associated with upregulation of invasion- and inflammation-promoting soluble factors, such as matrix metalloproteinase 2 (MMP2), its activator MMP14, tissue inhibitors of matrix metalloproteinase 2 (TIMP2), chemokine ligand 2 (CCL2), and urokinase-type plasminogen activator (uPA), the latter two being linked to the recruitment of the monocytic cells. Intravenous injection of multipotent vascular wall-resident mesenchymal stromal cells (MSCs) counteracted lung inflammation and metastasis by an as yet unknown mechanism (Klein et al., 2016). Translation of the latter data to the clinical situation is difficult, since whole thorax irradiation of 15 gray (Gy) is not applied in radiotherapy. Nevertheless one should consider that induction of lung metastases in murine models does occur upon total body irradiation at doses as low as 0.3 Gy (Sofia Vala et al., 2010) and upon partial thorax irradiation at doses (10 Gy) (Feys et al., 2015) that can be received by the lungs during radiotherapy for neighboring organs such as the esophagus.

The pro-angiogenic effect on endothelial cells by the irradiation of cancer cells was demonstrated by the subcutaneous inoculation of cancer cells, irradiated or not, in mice (Chung et al., 2006) or onto the chick chorioallontoic membrane (CAM) (Parthymou et al., 2004) with evaluation of microvessel density. Increased angiogenesis was observed even when the growth of the cancer cells was reduced after a dose of $40 \mathrm{~Gy}$. In vitro coculture of endothelial cells with irradiated cancer cells in the Matrigel invasion assay without direct contact between both cell types pointed to a role for soluble mediators (Abdollahi et al., 2005). Implication of such mediators was confirmed using conditioned media from irradiated as compared to unirradiated cancer cells in assays for capillary tube formation (Kargiotis et al., 2008; Badiga et al., 2011), chemotactic migration (Parthymou et al., 2004) and Matrigel invasion (Kaliski et al., 2005). In contrast, conditioned medium from irradiated fibroblasts isolated from human lung cancer reduced transwell migration of human umbilical vein endothelial cells (HUVEC) and left tube formation on Matrigel unchanged (Hellevik et al., 2013). Irradiation of a Matrigel plug, a surrogate implant mimicking the primary tumor, 10 days after subcutaneous implantation in mice stimulated its colonization by capillaries. This finding was confirmed by the observation of enhanced infiltration of CD31-positive cells after local irradiation of Lewis lung carcinoma-bearing mice. Ex vivo irradiation-mediated enhancement of angiogenesis was demonstrated by capillary sprouting from arterioles isolated from these mouse tumors (Sonveaux et al., 2003). Also irradiation of glioma at 10 and 40 Gy on the chick CAM induces angiogenesis when administered $48 \mathrm{~h}$ after inoculation (Parthymou et al., 2004). After ablative doses, restoration of the vasculature in experimental glioblastoma by vasculogenesis is mediated by irradiation-induced influx of bone marrow derived monocytes and macrophages, which eventually leads to tumor regrowth (Kioi et al., 2010a; Russell \& Brown, 2013a; Wang et al., 2013). Normalization of the vasculature occurred after irradiation at a dose of 5 times 5 Gy of a subcutaneous colorectal tumor in a rat where dynamic contrast-enhanced magnetic resonance imaging with gadomelitol showed reduction of neovascular leakage, enhanced tissue oxygenation and enhanced expression of vascular endothelial growth factor (VEGF) (Ceelen et al., 2006).

Irradiation of endothelial cells revealed direct antiangiogenic as well as pro-angiogenic effects, which seems to be dependent on the irradiation dose. At doses between 2 and 15 Gy, irradiated HUVEC provoked acute (within $24 \mathrm{~h}$ ) apoptosis and inhibited survival, proliferation, capillary tube formation on Matrigel and invasion through Matrigel (Abdollahi et al., 2005; Albert et al., 2006; Imaizumi et al., 2010). Inhibition of subsequent angiogenesis from quiescent endothelial cells about 1 week after irradiation was demonstrated in various in vivo and in vitro assays (Imaizumi et al., 2010). A lower dose (0.5 and $0.8 \mathrm{~Gy}$ ) may exert a pro-angiogenic activity as shown by enhanced wound healing migration of HUVEC (Sofia Vala et al., 2010). Indeed, hypo-fractionated irradiation delivering more than 5 Gy per fraction destroys the microvasculature more efficiently than conventional irradiation (De Wolf et al., 2015). At biologically equivalent doses, photons stimulate migration of ECV304 human endothelial cells whereas carbon-ions work inhibitory. In line with this, capillary tube formation was abolished by carbonions at doses, at which it was conserved after photon irradiation (Takahashi et al., 2003).

Total body irradiation (TBI) or irradiation of host sites or sites of cancer cell implantation influenced spontaneous or cancer cell-induced angiogenesis. TBI of C57/BL mice at a high dose of 15 Gy inhibited ex vivo aortic ring formation (Imaizumi et al., 2010); at a dose smaller than 1 Gy, TBI enhanced splenocyte-induced angiogenesis in mice (Kaminski et al., 1983) and spontaneous angiogenesis in transgenic embryonic Zebra fish (Sofia Vala et al., 2010). Similarly, local irradiation was pro-angiogenic at a dose of 0.3 Gy (Sofia Vala et al., 2010) whereas higher 
doses, more relevant to the clinical situation, inhibited angiogenesis in the Matrigel plug assay (Imaizumi et al., 2010; Kuonen et al., 2012b). However, this inhibition of angiogenesis does not necessarily work anti-metastatic. In a model mimicking local breast cancer relapse after radiotherapy, irradiation (20 Gy) of the mouse mammary gland before injection of syngeneic AT1 cells reduced microvessel density, which was accompanied by enhanced local invasion and metastasis to lymph nodes, lungs and liver (Kuonen et al., 2012b).

Apart from the irradiation dose, the timeline also influences the angiogenic response. Implantation of C6 glioma cells on the chick CAM following irradiation entailed inhibition of vessel formation during the first hours; which was followed by attraction of new vessels within the next $48 \mathrm{~h}$ (Polytarchou et al., 2004).

The above summarized experiments provide arguments to accept that irradiation of cancer cells or of elements of the tumor microenvironment may support or destroy the tumor vasculature and that both support and destruction may enhance invasion and metastasis.

\section{Invasion}

Homotypic cell-cell adhesion The homotypic cell-cell adhesion molecule E-cadherin was identified as one of the first invasion-suppressors. Its downregulation engages epithelial mesenchymal transition (EMT) in embryonic development and in cancer (Mareel et al., 1993). Irradiation has been shown to induce EMT via the upregulation of integrin, MMP9 and MMP2 and the downregulation of E-cadherin and cytokeratin 19 (Rajput et al., 2015a), as reviewed in detail by Lee et al. (Lee et al., 2017a) Irradiation causes EMT in cancer cells from various origins, such as prostate (Chang et al., 2013a), esophagus (He et al., 2015a), lung (Ho et al., 2010a; Liu et al., 2014c; Zhou et al., 2012; Zhao et al., 2017), colorectum (Kawamoto et al., 2012; Timaner et al., 2015), breast (Rajput et al., 2015; Kim et al., 2015; Kuo et al., 2015a; Yuan et al., 2015a; Zhou et al., 2011a), uterine cervix (Yan et al., 2013a), stomach (Zhang et al., 2015b), and glioma (Park et al., 2012). This irradiation-induced EMT is also accompanied by increased cell migration and invasion (He et al., 2015a; Tsukamoto et al., 2007). Via interaction with multiple pathways, the transcription factors zinc finger protein SNAI1 (Snail) and zinc finger E-box-binding homeobo 1 (ZEB1) appears as key in this irradiation-induced EMT (Fig. 1) (Lee et al., 2017a). Phosphorylation by kinases and dephosphorylation by phosphatases play a role in the fine tuning of irradiation-modulated EMT. The serine-threonine kinase

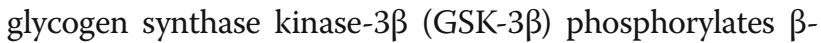
catenin so directing it to the ubiquitin proteasome degradation complex. Failure of GSK-3 $\beta$ leads to accumulation of $\beta$-catenin and translocation to the nucleus where it serves as a transcription factor in association with $\mathrm{T}$-cell factor (TCF). Downstream genes of the $\beta$-catenin/TCF complex include AXIN2 (encoding axis-inhibition protein 2), FZD7 (encoding Frizzled 7), HNF1A, CCND1 (encoding cyclin D1), CD44, GCSF (encoding granulocytecolony stimulating factor (G-CSF)), VEGF, MMP2, and MMP9, all implicated in invasion and metastasis. At the other side of the balance, the serine-threonine protein kinase tank-binding kinase-1 (TBK-1), a member of the inhibitor $\kappa B(\mathrm{I} \kappa \mathrm{B})$ kinase-related kinase family, activates GSK-3 $\beta$, in this way attenuating irradiation-induced EMT (Liu et al., 2014c). Fractionated irradiation using sublethal doses administered over a period of 2 months and clonal selection for radioresistant esophageal cancer cells leads to downregulation of the phosphatase and tensin homolog (PTEN) and upregulation of phosphoinositide 3-kinase (PI3K) which is linked with inactivation of GSK-3 $\beta$ and is associated with elevated Snail protein, which enhances EMT (He et al., 2015a). Furthermore, the activation of PI3K and subsequent stabilization of $\beta$ catenin in non-small-cell lung cancer cells, via the administration of 2 Gy on 3 consecutive days, promotes G-CSF, which in turn instigates EMT through JAK-STAT signaling pathways (Cui et al., 2015a).

Migration Migration of invading cells is executed through reorganization of the actin cytoskeleton and directed by the assembly/disassembly equilibrium of the cytoplasmic microtubule complex. By alteration of the biomechanical properties and the organization of the actin cytoskeleton, photon irradiation can promote invasion and migration of cancer cells, as observed in various cell lines via wound-healing assays, transwell migration assays and spheroid migration assays (see Table 2) (Kargiotis et al., 2008; Rajput et al., 2015a; Zhao et al., 2017; Kawamoto et al., 2012; Zhou et al., 2011a; Zhang et al., 2015b; Gu et al., 2015a; Tsutsumi et al., 2009; Nalla et al., 2010; De Bacco et al., 2011a; Fujita et al., 2011; Pickhard et al., 2011a; Fujita et al., 2012; Burrows et al., 2013; Ghosh et al., 2014; Murata et al., 2014; Fujita et al., 2015a; Nakayama et al., 2016; Fujita et al., 2014a; Fujita et al., 2015b; Zhai et al., 2006a; Zheng et al., 2015; Ohuchida et al., 2004). Carbon-ion irradiation can also stimulate invasion via the same PI3K pathway in certain cell lines. However, in several cell lines it was observed that carbon-ion irradiation inhibits migration and invasion via the ubiquitin-proteasome-mediated degradation of guanosine triphosphatase (GTP)-bound Rac1 and GTP-bound RhoA (Fujita et al., 2015a; Fujita et al., 2015b; Akino et al., 2009). Of note, in the majority of experiments migration and invasion were affected in the same direction by irradiation; however, an opposite effect was observed in two pancreas cancer cell lines (Qian et al., 2003) and in one breast cancer cell line (Rajput et al., 2015a) with hampered migration and improved 


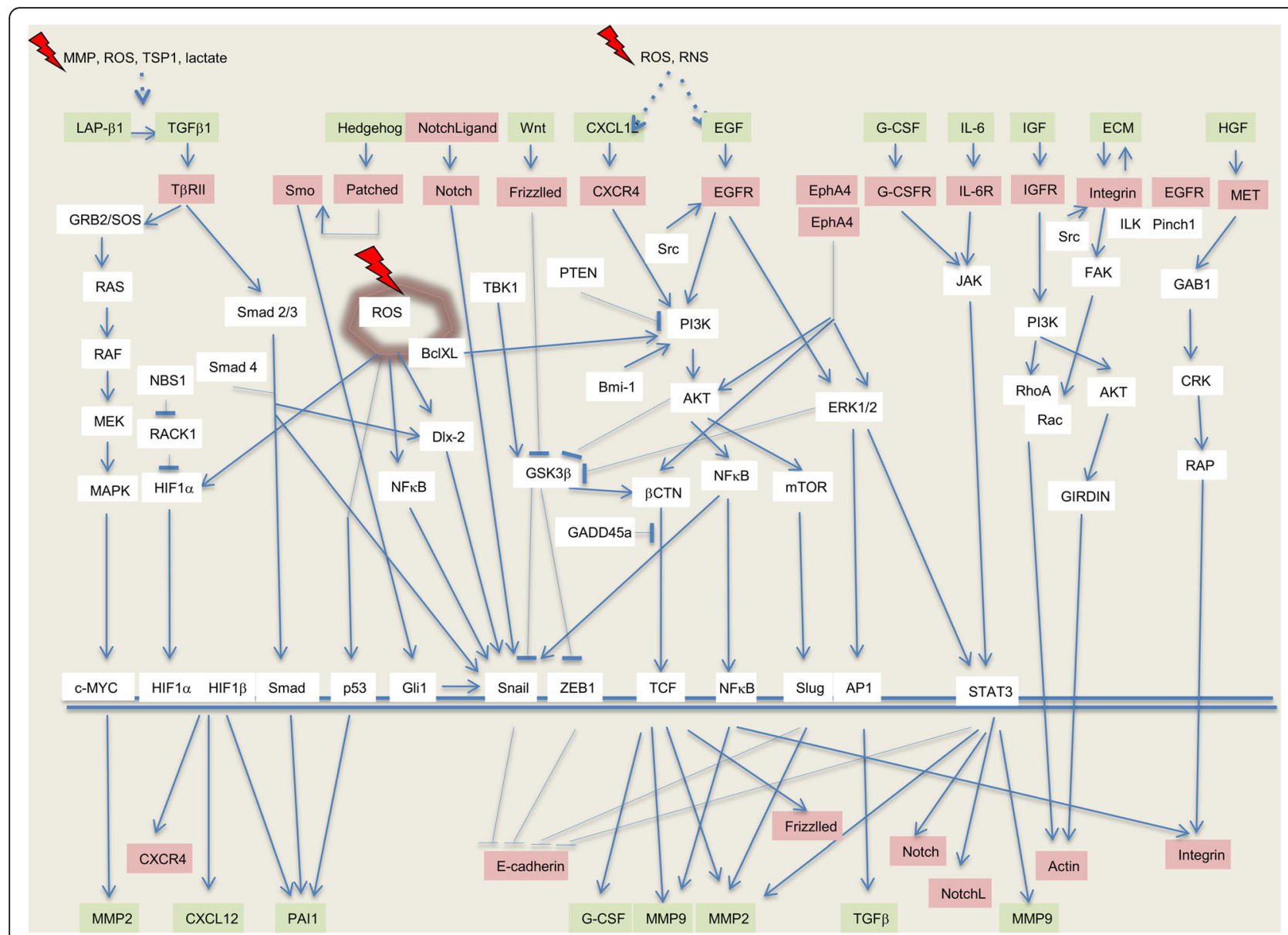

Fig. 1 Molecular pathways regulating cellular activities implicated in radiation -enhanced invasion and metastasis. Modified after Zhai et al., (2006b), Chargari et al., (2013), He et al., (2015b) and Lee et al., (2017b), with data from: Ahmed et al., (2013b); Asuthkar et al., (2011); Bastos et al., (2014); Chang et al., 2013b; Cheng et al., (2006b); Cho et al., (2016b); Cui et al., (2015b); de Marcondes (2017); Dong et al., (2015b); Eke \& Cordes, (2015b); Fujita et al., (2014b); Fujita et al., (2015c); Gu et al., (2015b); He et al., (2015b); Ho et al., (2010b); Kang et al., (2013); Yan et al., (2013b); Kim et al., (2016); Kuo et al., (2015b); Liu et al., (2014b); Park et al., (2006b); Pichard et al., (201 1b); Rajput et al., (2015b); Yuan et al., (2015b); Zhai et al., (2006b); Zhou et al., (201 1b). Green, red and white boxes are secretory, transmembrane receptor and intracellular signaling molecules respectively. The glowing box is a mitochondrion; the double line is DNA. Abbreviations: Akt, protein kinase B; AP1, activator protein 1; BclxL, B-cell lymphoma-extra large; Bmi-1, B cellspecific Moloney murine leukemia virus integration site 1; $\beta$-CTN, beta-catenin; CRK, CT10 regulator of kinase; CXCL12, C-X-C motif chemokine 12; CXCR4, C-X-C chemokine receptor type 4; Dlx-2, distal-less homeobox-2; ECM, extracellular matrix; EGF, epidermal growth factor; EGFR, epidermal growth factor receptor; EphA4, ephrin A 4; ERK, extracellular signal-regulated kinase; FAK, focal adhesion kinase; GAB1, GRB2-associated-binding protein; GADD45a, Growth Arrest and DNA Damage inducible Alpha; GCSF, granulocyte colony stimulating factor; GCSFR, granulocyte colony stimulating factor receptor; GIRDIN, Guanine nucleotide-binding protein a subunit -interacting vesicle-associated protein; Gli1, Glioma-associated oncogen; GRB2, Growth factor receptor-bound protein 2; GSK3 $\beta$, glycogen synthase kinase 3 beta; HGF, hepatocyte growth factor; HIF-1, hypoxia-inducible factor-1; IGF, insulinlike growth factor; IGFR, insulin-like growth factor receptor; IL-6, interleukin 6; ILK, integrin-linked kinase; JAK, Janus kinase; LAP- $\beta 1$, latency-associated peptide of TGF- $\beta$; MEK, mitogen-activated kinase kinase; MET, tyrosine-protein kinase Met; MMP, matrix metalloproteinase; mTOR, mechanistic target of rapamycin; NF-KB, nuclear-factor kappa-light-chain-enhancer of activated B cell; Nrf2, Nuclear factor E2 related factor 2; NSB1, Nijmegen breakage syndrome 1; p53, Tumor protein p53; PAl-1, plasminogen activator inhibitor 1; PAK1, p21- activated kinase 1; PI3K, phosphatidylinositol 3-kinase; PTEN, phosphatase and tensin homolog; Rac, Ras-related C3 botulinum toxin substrate; RACK1, receptor for activated C kinase 1; RAF, Rapid Accelerated Fibrosarcoma; RAP, Ras-proximate; RAS, Rat sarcoma; RhoA, Ras homolog gene family member A; RNS, reactive nitrogen species; ROS, reactive oxygen species; Src, sarcoma family kinase; SMAD, small and mothers against decapentaplegic; Smo, Smoothened; SOS, Son of Sevenless; STAT, signal transducer and activator of transcription; TCF, T-cell factor; TGF- $\beta$, transforming growth factor beta; TKB-1, tank-binding kinase-1; TSP1, Trombospondin 1; T $\beta R$ Rl, TGF- $\beta$ type II receptor; Wnt, Wingless-related integration site; ZEP1, Zinc finger E-box-binding homeobox 1

invasion. Irradiation-enhanced invasion was also shown in organ culture where normal brain tissue fragments were confronted with aggregates of cancer cells, mimicking local invasion as it occurs in gliomas (Wick et al., 2002; Wild-Bode et al., 2001).
Various elements of the ecosystem served as successful targets for irradiation-mediated enhancement of invasion. Cancer cells, mostly issued from cell lines, were target in most studies (Table 2); host cells were targets in some experiments. For instance, irradiation of reconstituted 
Table 2 Effect of radiation on the invasion of cancer cells in Matrigel-coated two-compartment chambers in vitro

\begin{tabular}{|c|c|c|c|c|c|c|c|}
\hline \multicolumn{2}{|l|}{ Cancer cells } & \multicolumn{2}{|l|}{ Radiation } & \multirow{2}{*}{$\begin{array}{l}\text { Invasion } \\
\text { IR/Ctrl }\end{array}$} & \multirow[t]{2}{*}{ Molecular } & \multirow[t]{2}{*}{ Inhibitor } & \multirow[t]{2}{*}{ Ref } \\
\hline Origin & Code & Mode & Dose & & & & \\
\hline \multirow[t]{8}{*}{ Glioblastoma } & U87MG; LN-18; LN-229 & ${ }^{137} \mathrm{Cs}$ & 6 Gy & $5 ; 3 ; 3.7$ & MMP; BCL-2 & & (Wild-Bode et al., 2001) \\
\hline & LN-229; U87MG & ${ }^{137} \mathrm{Cs}$ & $6 \mathrm{~Gy}$ & $1.5 ; 1.7$ & $\operatorname{av} \beta 3$ integrin & temozolomide & (Wick et al., 2002) \\
\hline & A-172; U-138 & $240 \mathrm{kV}$ & $8 \mathrm{~Gy}$ & $0.07 ; 1$ & & & (Cordes et al., 2003) \\
\hline & $\begin{array}{l}\text { U251; U373; LN18; } \\
\text { LN428 }\end{array}$ & ${ }^{137} \mathrm{CS}$ & 5 Gy & $1.5 ; 1$ & EGFR/SrC & & (Park et al., 2006a) \\
\hline & UN3; GM2 & $\mathrm{nm}$ & 6 Gy & $2.2 ; 2.2$ & IGF-1/Rho & & (Zhai et al., 2006a) \\
\hline & U87 & MV & 3 Gy & 1.7 & Wnt/ßCTN & XAV939 & (Dong et al., 2015a) \\
\hline & U251 & $200 \mathrm{kV}$ & 10 Gy & 1.3 & MMP/TIMP & Paputilone & $\begin{array}{l}\text { (Furmanova-Hollenstein } \\
\text { et al., 2013) }\end{array}$ \\
\hline & $C 6 L^{*}$ & ${ }^{137} \mathrm{Cs}$ & 3 Gy & 2 & EMT markers & & (Park et al., 2012) \\
\hline Meduloblastoma & DA0Y; D283 & RS 2000 & 7 Gy & $1.5 ; 1.5$ & UPAR; $\beta 1 / F A K$ & & (Nalla et al., 2010) \\
\hline Neuroblastoma & $\begin{array}{l}\text { SH-EP; SK-N-SH; SH-SY5Y; } \\
\text { SK-N-AS; NLF }\end{array}$ & $200 \mathrm{kV}$ & $6 \mathrm{~Gy}$ & $4 ; 2.5 ; 4 ; 1 ; 1$ & HGF/Met & & (Schweigerer et al., 2005) \\
\hline Meningioma & IOMM-Lee & $150 \mathrm{kV}$ & 5 Gy & 1.8 & UPAR & & (Kargiotis et al., 2008) \\
\hline \multirow[t]{6}{*}{ Pancreatic Ca } & Panc-1; Suit-2; Hs766T & ${ }^{137} \mathrm{Cs}$ & $10 \mathrm{~Gy}$ & $2.0 ; 1.6 ; 1$ & MMP-2 & CGS27023A & (Qian et al., 2002) \\
\hline & Panc-1; AsPC-1; Suit-2 & ${ }^{137} \mathrm{Cs}$ & $10 \mathrm{~Gy}$ & $1.5 ; 1.3 ; 1.4$ & HGF/Met & NK4 & (Qian et al., 2003) \\
\hline & $\begin{array}{l}\text { PANC-1; AsPC-1; BxPC-3; } \\
\text { MIAPaCa-2 }\end{array}$ & C-ion & 2 Gy & $4 ; 0.5 ; 0.3 ; 0.1$ & MMP2 & GM6001 & (Fujita et al., 2012) \\
\hline & PANC-1 & C-ion & 2 Gy & 2.5 & $\mathrm{NO} / \mathrm{PI} 3 \mathrm{~K}$ & Table 1 & (Fujita et al., 2014a) \\
\hline & $\begin{array}{l}\text { AsPC-1, BxPC-3, } \\
\text { MIAPaCa-2 }\end{array}$ & C-ion & 2 Gy & 0.01 & Rac1/RhoA & & (Fujita et al., 2015a) \\
\hline & MIA-PaCa; PANC-1 & X-ray & 4 Gy & $3 ; 1.6$ & MMP-2 & GM6001 & (Fujita et al., 2011) \\
\hline \multirow[t]{2}{*}{ Lung SCC } & EBC-1 & C-ion & 2 Gy & 0.6 & & & (Akino et al., 2009) \\
\hline & EBC-1 & $4 \mathrm{MV}$ & 2 Gy & 1.2 & & & (Akino et al., 2009) \\
\hline \multirow[t]{12}{*}{ Lung Adenoca } & A549 & C-ion & 2 Gy & 0.4 & ANLN & & (Akino et al., 2009) \\
\hline & A459 & $4 \mathrm{MV}$ & 2 Gy & 0.5 & & & (Akino et al., 2009) \\
\hline & A459 & ${ }^{137} \mathrm{Cs}$ & $10 \mathrm{~Gy}$ & 2.5 & BCl-xl/STAT3 & & (Ho et al., 2010a) \\
\hline & A549 & C-ion & 10 Gy & 0.2 & PI3K Akt & & (Ogata et al., 2011) \\
\hline & & $4 \mathrm{Mv}$ & $10 \mathrm{~Gy}$ & 0.5 & & & \\
\hline & A549 & ${ }^{60} \mathrm{Co}$ & $2 \mathrm{~Gy}$ & $\times 1.5$ & MMPs & & (Zhou et al., 2012) \\
\hline & A459; H1299 & MV & 4 Gy & $0.7 ; 0.7$ & TBK1 & MG132 & (Liu et al., 2014c) \\
\hline & A549; HT1299 & ${ }^{137} \mathrm{Cs}$ & $3 \times 2$ Gy & $1.5 ; 1.5$ & G-CSF & & (Cui et al., 2015a) \\
\hline & H1299; A459; H460 & MV & 2 Gy & $1.5 ; 2 ; 1.2$ & CXCR4 & & (Gu et al., 2015a) \\
\hline & A549 & ${ }^{137} \mathrm{Cs}$ & 10 Gy & $\times 1.8$ & EGFR & Gefitinib; PPA & (Cho et al., 2016a) \\
\hline & A549; H460 & X-ray & 4 Gy & $\times 1.5 ; \times 1.4$ & Nrf2/Notch & & $\begin{array}{l}\text { (Parthymou et al., 2004; } \\
\text { Zhao et al., 2017) }\end{array}$ \\
\hline & LLC-LM* & ${ }^{60} \mathrm{Co}$ & 7,5 Gy & $\times 3,5$ & MMP9 & & (Chou et al., 2012) \\
\hline \multirow[t]{8}{*}{ Mammary Ca } & MCF7 & ${ }^{60} \mathrm{Co}$ & 2 Gy & $\times 1.4$ & TGF- $\beta$ & & (Zhou et al., 2011a) \\
\hline & MCF7; SKBR3 & ${ }^{137} \mathrm{Cs}$ & $3 \times 2$ Gy & $\times 5 ; 4.3$ & SRC/PI3K & & (Kim et al., 2015) \\
\hline & MCF7 & ${ }^{137} \mathrm{Cs}$ & 5 Gy & $\times 2$ & NBS1/HIF-1a & & (Kuo et al., 2015a) \\
\hline & MCF7; MDA-MB-231 & & 5 Gy & 1.7 & TGF- $\beta$ & Thymoquinone & (Rajput et al., 2015a) \\
\hline & MDA-MB-231 & & 5 Gy & $\times 2$ & & & (Vilalta et al., 2014) \\
\hline & MCF7 & ${ }^{137} \mathrm{Cs}$ & 2 Gy & $\times 1.6$ & EMT & histamine & (Galarza et al., 2016) \\
\hline & MDA-MB-231, & $6 \mathrm{MV}$ & $10 \mathrm{~Gy}$ & $\times 2$ & Met & PHA665752 & (De Bacco et al., 2011a) \\
\hline & MCF-7 & X-ray & $20 \times 1$ Gy & $\times 5$ & Snail; twist & & (Zhang et al., 2011) \\
\hline
\end{tabular}


Table 2 Effect of radiation on the invasion of cancer cells in Matrigel-coated two-compartment chambers in vitro (Continued)

\begin{tabular}{|c|c|c|c|c|c|c|c|}
\hline \multicolumn{2}{|l|}{ Cancer cells } & \multicolumn{2}{|c|}{ Radiation } & \multirow{2}{*}{$\begin{array}{l}\text { Invasion } \\
\text { IR/Ctr| }\end{array}$} & \multirow[t]{2}{*}{ Molecular } & \multirow[t]{2}{*}{ Inhibitor } & \multirow[t]{2}{*}{ Ref } \\
\hline Origin & Code & Mode & Dose & & & & \\
\hline & $4 T 1^{*}$ & ${ }^{137} \mathrm{Cs}$ & $20 \mathrm{~Gy}$ & $\times 3.5$ & GM-CSF & & (Vilalta et al., 2014) \\
\hline Prostatic Ca & PC-3; DU145 & $6 \mathrm{MV}$ & $5 \times 2 \mathrm{~Gy}$ & $\times 2$ & PI3K/AKT & BEZ235 & (Chang et al., 2013) \\
\hline Oral Ca & OECM1 & ${ }^{137} \mathrm{Cs}$ & 5 Gy & $\times 2.4$ & & & (Kuo et al., 2015a) \\
\hline \multirow{2}{*}{$\begin{array}{l}\text { Esophageal. } \\
\text {.SCC }\end{array}$} & KYSE-150 & $150 \mathrm{kV}$ & 37 Gy & $\times 1.3$ & PTEN & & (He et al., 2015a) \\
\hline & TE-9 & $125 \mathrm{kV}$ & 2 Gy & 15 & TGF- $\beta$ & metformin & (Nakayama et al., 2016) \\
\hline Hepatoma & HepG2; Huh7; & ${ }^{60} \mathrm{Co}$ & $7.5 \mathrm{~Gy}$ & $\times 3$ & PI3K & & (Cheng et al., 2006a) \\
\hline \multirow[t]{3}{*}{ Colon Ca } & CaR1; DLD1 & $100 \mathrm{kV}$ & 5 Gy & $6.5 ; 4.3$ & EMT & & (Kawamoto et al., 2012) \\
\hline & HCT116 & ${ }^{137} \mathrm{Cs}$ & 4 Gy & $\times 1.7$ & MMP & & (Speake et al., 2005) \\
\hline & HT29 & ${ }^{137} \mathrm{Cs}$ & 5 Gy & 2 & $\beta C T N / T C F$ & & (Bastos et al., 2014) \\
\hline Cervical Ca & Siha; C33A & X-ray & 75 Gy & $\times 3 ; 3$ & NF-kB p65 & & (Yan et al., 2013a) \\
\hline \multirow[t]{5}{*}{ Fibrosarcoma } & HT180 & proton & 2 Gy & 0.2 & MMP-2 & & (Ogata et al., 2005) \\
\hline & & C-ion & 2 Gy & 0.2 & MMP-2 & & \\
\hline & & $4 \mathrm{MV}$ & 2 Gy & $\times 1.6$ or 1 & MMP-2 & GM6001 & \\
\hline & HT1080 & ${ }^{137} \mathrm{Cs}$ & 4 Gy & $\times 1.5$ & MMP- $2 \& 9$ & & (Speake et al., 2005) \\
\hline & HT180 & $200 \mathrm{kV}$ & 2 Gy & $\times 1.5$ & MMP/TIMP & patupilone & $\begin{array}{l}\text { (Furmanova-Hollenstein } \\
\text { et al., 2013) }\end{array}$ \\
\hline Melanoma & $\mathrm{B} 16^{*}$ & ${ }^{137} \mathrm{Cs}$ & 8 Gy & $\times 3$ & MMP-2 & Metastat & (Kaliski et al., 2005) \\
\hline
\end{tabular}

Most cell lines are human, except cell lines marked by an asterisks which are rodent. Inhibitors comprise agents that were used clinically, namely: BEZ235, an imidazoquinolone derivative and an inhibitor of PI3K and mTOR; CGS27023A, a matrix metalloproteinase inhibitor and anti-metastatic agent; Gefitinib, tyrosine kinase inhibitor targeting EGFR; GM6001, a broad -spectrum matrix metalloproteinase inhibitor known as galardin; Histamine, a biogenic amine that targets four (H1-H4) histamine subtypes G-protein coupled receptors (GPCR); Metastat, a chemically modified tetracycline and inhibitor of gelatinase and MMP; Metformin, an anti-diabetic; MG132, a specific proteasome inhibitor; NK4, a four-kringle antagonist of hepatocyte growth factor and an angiogenesis inhibitor; Patupilone, a microtubule stabilizing drug; PHA665752, an ATP-competitive small-molecule inhibitor of the tyrosine kinase c-Met; PPA, Podophyllotoxin acetate, a microtubule inhibitor and a spindle poison; temozolomide, an alkylating chemotherapeutic drug; Thymoquinone, a regulator of NF- kB and extracellular signal-regulated kinase (ERK) signaling pathways. Abbreviations: Akt, protein kinase B; ANLN, human homologue of anailin; b1, beta1 integrin subunit, b-CTN, beta-catenin; BcL-2, B-cell lymphoma 2; BclxL, B-cell lymphoma-extra large; C-ion, carbon-ions; Ca, carcinoma; Co, cobalt; Cs, cesium; Ctrl, unirradiated control; CXCR4, C-X-C chemokine receptor type 4; EGFR, epidermal growth factor receptor; EMT, epithelial-to-mesenchymal transition; FAK, focal adhesion kinase; GCSF, granulocyte colony stimulating factor; Gy, gray; HGF, hepatocyte growth factor; IGF1, insulin-like growth factor-1; Met, tyrosine-protein kinase Met; MMP, matrix metalloproteinases; nm, not mentioned; NO, nitric oxide; Nrf2, Nuclear factor E2 related factor 2; PI3K, phosphatidylinositol 3-kinase; PPA, Podophyllotoxin acetate; Rho, Ras homolog gene family member; SCC: squamous cell carcinoma; Src,sarcoma family kinase; STAT, signal transducer and activator of transcription; TBK1, tank-binding kinase 1; TCF, T-cell factor; TGFb, transforming growth factor beta; TIMP, tissue inhibitor of matrix metalloproteinase; TGF-b, transforming growth factor beta; uPAR, urokinase-type plasminogen activator receptor; Wnt, Wingless-related integration site; XAV 939, a selective $\beta$-catenin-mediated transcription inhibitor

vascular wall led to increased degradation by unirradiated HT1080 sarcoma cells (Heisel et al., 1984). Irradiated EA. hy926 endothelial cells enhanced the migration of human MDA-MB-231 and murine 4 T1 breast cancer cells (Feys et al., 2015). Enhanced colon cancer cell adhesion to HUVEC was obtained when both elements were irradiated (Hamalukic et al., 2011a). Variations in the elements of the ecosystems and of the experimental manipulations influenced irradiation-enhancement of invasion-related cellular activities, though various authors did not always obtain the same result.

The invasive response to irradiation seems to be dosedependent. Dose-response curves were registered for single doses between 1 Gy and 10 Gy (Burrows et al., 2013; Park et al., 2006a). Maximal enhancement of invasion-related activities was obtained at single doses around 4 to 5 Gy (Park et al., 2012; Fujita et al., 2011). In most of these experiments with sublethal doses, proliferation was inhibited, a situation that is known to be permissive for migration and invasion (Storme \& Mareel, 1984). Proliferation was unchanged in human glioma cells at the lower dose of 20 cGy that led to enhanced wound-healing migration (Ghosh et al., 2014). To obtain more invasive radioresistant cell lines, doses varied largely: $1 \times 6$ Gy (Su et al., 2012); $1 \times 10$ Gy (Tsutsumi et al., 2009); $5 \times 2$ Gy daily (Chang et al., 2013); $20 \times 1$ Gy (Zhang et al., 2011) and $35 \times 2$ Gy (He et al., 2015a; Yan et al., 2013a). In the experiments by Zhang et al. cells were tested shortly after irradiation, in contrast to the others where irradiation occurred weeks before testing in the in vitro ecosystem as they were maintained and selected to obtain resistant cell lines (Zhang et al., 2011). The pro-invasive effect of $10 \mathrm{~Gy}$ on lung adenocarcinoma cells was transient with enhancement after 16 and $24 \mathrm{~h}$ but not after 48 and $72 \mathrm{~h}$ (Ho et al., 2010a).

As to the organ of origin, Fujita et al. compared 31 cell lines irradiated or not with a single dose and found qualitative (e.g. stimulation of inhibition of invasion) and 
quantitative (e.g. the extent of stimulation) differences in their invasive response, that were not related to their organ of origin (Fujita et al., 2015b). In the same experimental series, differences in invasive response to irradiation were found between cancer cells of similar organ of origin, namely glioma (Cordes et al., 2003), lung (Akino et al., 2009), thyroid (Burrows et al., 2013), and breast cancer (Paquette et al., 2011). Opposite effects of irradiation were described for the same cell lines also by different authors, e.g. MCF7 breast cancer cells (Kim et al., 2015; Paquette et al., 2011; Galarza et al., 2016), pancreatic cancer cells (Fujita et al., 2012; Qian et al., 2002), glioblastoma cells (Wick et al., 2002; Wild-Bode et al., 2001; Cordes et al., 2003) and lung cancer cells (Zhao et al., 2017; Zhou et al., 2011a; Gu et al., 2015a; Jung et al., 2007; Cho et al., 2016a; Ogata et al., 2011; Liu et al., 2014a). Published details of these experiments did not permit us to conclude which variables were responsible for such opposite results. With regard to the mode of irradiation, no systematic differences were noted between photons with lower $(250 \mathrm{kV})$ as compared to higher (MV) energy (Table 2). Heavy particles (carbon-ions) were clearly different in as much as they inhibited invasion at biologically equivalent or lower doses that lead to enhancement with photons (Fujita et al., 2012; Fujita et al., 2015a; Fujita et al., 2015b). This was also demonstrated in matched experiments using the same cell types and the same cellular activities as an endpoint (Ogata et al., 2005; Rieken et al., 2012; Stahler et al., 2013), though there are exceptions that showed stimulation (Murata et al., 2014) or inhibition (Akino et al., 2009; Ogata et al., 2011) for both modes of irradiation.

Epidermal growth factor receptor (EGFR)-positive breast cancer cells, but not negative ones, were induced to invade by ionizing radiation in a reactive oxygen species (ROS)-dependent manner (Kambach et al., 2014). In human lung adenocarcinoma cells (A549), ROS function as intracellular second messengers that participate in the activation of intracellular signaling pathways and, therefore, may mediate ligand-independent and rapid (within $5 \mathrm{~min}$ ) phosphorylation of cell surface receptors, like EGFR (Lee et al., 2008). Kinetic analysis showed that the non-receptor kinase Src was transiently activated within 30 min of irradiation at a dose of 10 Gy, whereas ErbB2 phosphorylation began to increase after $60 \mathrm{~min}$ and was maintained for at least $24 \mathrm{~h}$. The signal transducer and activator of transcription factor 3 (STAT3) was observed to be phosphorylated also $30 \mathrm{~min}$ after irradiation, preceding the accumulation of the $\mathrm{Bcl}-2$ anti-apoptotic family member $\mathrm{B}$ cell lymphoma-extra large $\left(\mathrm{Bcl}-\mathrm{X}_{\mathrm{L}}\right)$ and stimulation of invasion (Table 2) (Ho et al., 2010a). In the latter experiments, applying a single exposure of 10 Gy, irradiation-induced STAT3 phosphorylation was not stable, and decreased with continued incubation, reflecting a downregulation of malignant signals. Indeed, $\mathrm{Bcl}-\mathrm{X}_{\mathrm{L}}$ and EMT markers were restored to their original levels when incubation continued for up to 48-72 $\mathrm{h}$ and invasion did no longer differ from that of unirradiated controls. It would be interesting to know whether or not this reversibility could be overcome by a multiple exposures as it is applied in fractionated radiotherapy. Curiously and in line with the results on invasion, carbon-ions do not affect EGFR activity or the downstream signaling effectors (Fujita et al., 2015b; Stahler et al., 2013).

Cell-matrix adhesion Cell-matrix adhesion is mediated by transmembrane receptors, called integrins that bind to the extracellular matrix and signal intracellularly. Integrins contribute to invasion and metastasis by regulating cell motility, localization and activity of MMPs, as well as cell survival (Desgrosellier \& Cheresh, 2010). Integrins are linked to the EGFR through integrin-linked kinase (ILK) and particularly interesting new cysteine-histidine-rich 1 (PINCH1) forming multiprotein and multifunctional complexes cooperatively promoting invasion ( $\mathrm{Li}$ et al., 2013; Eke \& Cordes, 2015a). At their extracellular end $\alpha v \beta 5$ integrins cooperate with the matricellular protein cysteine-rich angiogenic inducer 61 (CYR61) mediating the pro-metastatic effect of tumor bed irradiation (Monnier et al., 2008). In vitro and in vivo experiments (Tables 2 and 3) show that invasion-enhancing doses of irradiation upregulate integrins as well as phosphorylate focal adhesion kinase (FAK) and paxillin, which are critical mediators of cell migration (Tsutsumi et al., 2009; Nalla et al., 2010; Gogineni et al., 2011). In pancreatic cancer cells upregulation of integrins was ascribed to increased transcription and to increased postendocytic recycling (Yao et al., 2011). A striking correlation between glioma cell migration and upregulation of $\alpha \mathrm{v} \beta 5$ integrin is described by Rieken et al. (Rieken et al., 2011) In contrast to photons, carbonions impaired migration through down-regulation of cell surface integrin expression. Also MMPs are activated upon irradiation-enhanced integrin expression, suggesting an interplay between the mesenchymal form of migration and cell matrix degradation (Wild-Bode et al., 2001; Yao et al., 2011; Vehlow \& Cordes, 1836). A forward feedback survival pathway was discovered in radioresistant breast cancer cells, where irradiationstimulated $\beta 1$ integrin causes NF- $\mathrm{\kappa B}$ to bind to the promoter region of $\beta 1$ integrin and to transactivate it (Ahmed et al., 2013a).

Proteolysis Breakdown of extracellular matrix (ECM) by invasive cells is mediated by lytic enzymes such as MMP2, MMP9 and membrane-bound MMP-1 (MT1MMP). These enzymes act in balance with inhibitors 
Table 3 Effect of radiation on local invasion or metastasis of rodent and human cancer cell lines as tested in syngeneic and in xenogeneic mice respectively

\begin{tabular}{|c|c|c|c|c|c|c|c|c|}
\hline \multicolumn{3}{|c|}{ Radiation } & \multicolumn{3}{|l|}{ Inoculum } & \multirow[t]{2}{*}{ Score $(\mid r / C t r l)$} & \multirow[t]{2}{*}{ Molecular } & \multirow[t]{2}{*}{ Ref } \\
\hline Target & Mode & Dose & Code & Origin & Site & & & \\
\hline & & & & Syngeneic & & & & \\
\hline \multicolumn{9}{|c|}{ Cancer cells } \\
\hline & ${ }^{137} \mathrm{Cs}$ & 3 Gy & $9 \mathrm{~L}$ & glioma & intracaranial & $40^{\mathrm{d}}$ & $\operatorname{av} \beta 3$ & (Wild-Bode et al., 2001) \\
\hline & C-ion & 5 Gy & LM8 & osteosarcoma & subcutaneous & 0.6 & & (Ogata et al., 2005) \\
\hline & C-ion & $5 \mathrm{~Gy}$ & LM8 & osteosarcoma & intravenous & 0.4 & & (Ogata et al., 2005) \\
\hline & $4 \mathrm{MV}$ & $10 \mathrm{~Gy}$ & LM8 & osteosarcoma & subcutaneous & 1 & & (Ogata et al., 2005) \\
\hline & $4 \mathrm{MV}$ & $10 \mathrm{~Gy}$ & LM8 & osteosarcoma & intravenous & 0.4 & & (Ogata et al., 2005) \\
\hline & $\mathrm{nm}$ & 3 Gy & $9 \mathrm{~L}$ & glioma & intracaranial & $2.4^{d}$ & |GFR1 & (Zhai et al., 2006a) \\
\hline \multicolumn{9}{|l|}{ CAF } \\
\hline & ${ }^{137} \mathrm{Cs}$ & 10 Gy & Suit_2/CAF & pancreatic ca & intrapancreas & $2.5^{\mathrm{d}}$ & Met & (Ohuchida et al., 2004) \\
\hline & $\mathrm{nm}$ & 4 Gy & PC/CAFCM & Pancreatic ca & intravenous & 4 & CXCL12 & (Li et al., 2016a) \\
\hline \multicolumn{9}{|c|}{ Mammary f. p. } \\
\hline & MV & $4 \times 6$ Gy & $\mathrm{D} 2 \mathrm{~A} 1 / \mathrm{FUCCl}$ & mammary ca & mammary f.p. & $1.8^{\mathrm{d}}$ & COX2; IL6 & (Bouchard et al., 2013) \\
\hline & MV & $4 \times 6$ Gy & $\mathrm{D} 2 \mathrm{~A} 1 / \mathrm{FUCCl}$ & mammary ca & mammary f.p. & $2^{c}$ & & (Bouchard et al., 2013) \\
\hline & MV & $4 \times 6$ Gy & $\mathrm{D} 2 \mathrm{~A} 1 / \mathrm{FUCCl}$ & mammary ca & mammary f.p. & 2.4 & & (Bouchard et al., 2013) \\
\hline & MV & $4 \times 6$ Gy & $\mathrm{D} 2 \mathrm{~A} 1 / \mathrm{FUCCl}$ & mammary ca & intravenous & 1 & & (Bouchard et al., 2013) \\
\hline & $125 \mathrm{kV}$ & $20 \mathrm{~Gy}$ & AT1 & mammary ca & mammary f.p. & $6^{d}$ & $\mathrm{HIF/KITL}$ & (Kuonen et al., 2012b) \\
\hline & $125 \mathrm{kV}$ & 20 Gy & AT1 & mammary ca & mammary f.p. & $8^{b}$ & & \\
\hline & $125 \mathrm{kV}$ & 20 Gy & AT1 & mammary ca & mammary f.p. & 6 & & \\
\hline \multicolumn{9}{|l|}{ Thorax } \\
\hline & $300 \mathrm{kV}$ & 10 Gy & MMT/PyVmT & mammary ca & intravenous & 4 & TGF $\beta$ & (Biswas et al., 2007) \\
\hline & $300 \mathrm{kV}$ & $10 \mathrm{~Gy}$ & MMT/PyVmT & mammary ca & mammary f.p. & $17^{c}$ & TGF $\beta$ & (Biswas et al., 2007) \\
\hline & $300 \mathrm{kV}$ & $10 \mathrm{~Gy}$ & MMT/PyVmT & mammary ca & mammary f.p. & 17 & TGF $\beta$ & (Biswas et al., 2007) \\
\hline & ${ }^{60} \mathrm{Co}$ & $1 \times 15$ Gy & TS/A & mammary ca & intravenous & 2.5 & & (Klein et al., 2016) \\
\hline & ${ }^{60} \mathrm{Co}$ & 1 X15 Gy & $\mathrm{B} 16 / \mathrm{F} 10$ & melanoma & intravenous & 2.3 & uPA;CCL2 & \\
\hline \multicolumn{9}{|c|}{ Partial lung } \\
\hline & $220 \mathrm{kV}$ & $10 \mathrm{~Gy}$ & $4 \mathrm{~T} 1$ & mammary ca & mammary f.p. & 3 & & (Feys et al., 2015) \\
\hline \multicolumn{9}{|c|}{ Abdomen } \\
\hline & $6 \mathrm{MeV}$ & $2 \mathrm{~Gy}$ & CT26 & colon ca & intravenous & $>1$ & & (Timaner et al., 2015) \\
\hline \multicolumn{9}{|c|}{ Total Body } \\
\hline & ${ }^{60} \mathrm{Co}$ & 10 Gy & B16/F10 & melanoma & subcutaneous & 2 & & (Klein et al., 2016) \\
\hline \multicolumn{9}{|l|}{ Tumor } \\
\hline & $250 \mathrm{kV}$ & $10 \times 0.6 \mathrm{~Gy}$ & KLN-205 & sarcoma & subcutaneous & $1^{a}$ & & (Baker et al., 1981) \\
\hline & ${ }^{137} \mathrm{Cs}$ & $5 \times 10$ Gy & LLC-LM & lung ca & subcutaneous & 10 & & (Camphausen et al., 2001) \\
\hline & ${ }^{137} \mathrm{Cs}$ & $5 \times 10 \mathrm{~Gy}$ & T241 & fibrosarcoma & subcutaneous & 14 & angiosatin & (Camphausen et al., 2001) \\
\hline & $6 \mathrm{MV}$ & $8 \mathrm{~Gy}$ & ALT-S1C1 & glioma & intracranial & 0.7 & & (Wang et al., 2013) \\
\hline & ${ }^{60} \mathrm{Co}$ & $5 \times 10$ Gy & LLC-M & lung ca & subcutaneous & 5 & MMP9 & (Chou et al., 2012) \\
\hline & & & & Xenogeneic & & & & \\
\hline \multicolumn{9}{|c|}{ Cancer cells } \\
\hline & ${ }^{60} \mathrm{Co}$ & $7.5 \mathrm{~Gy}$ & HepG2 & Hepatoma & intrahepatic & $3^{d}$ & MMP9 & (Cheng et al., 2006a) \\
\hline & ${ }^{60} \mathrm{Co}$ & 4 Gy & $\mathrm{CHO} 1$ & Fibrosarcoma & intravenous & 5 & & (Hamalukic et al., 2011a) \\
\hline & MV & $6 \mathrm{~Gy}$ & CaSki & Cervical ca & intravenous & 1.6 & Ras & (Su et al., 2012) \\
\hline
\end{tabular}


Table 3 Effect of radiation on local invasion or metastasis of rodent and human cancer cell lines as tested in syngeneic and in xenogeneic mice respectively (Continued)

\begin{tabular}{|c|c|c|c|c|c|c|c|c|}
\hline \multicolumn{3}{|c|}{ Radiation } & \multicolumn{3}{|l|}{ Inoculum } & \multirow[t]{2}{*}{ Score $(\mid r / C t r l)$} & \multirow[t]{2}{*}{ Molecular } & \multirow[t]{2}{*}{ Ref } \\
\hline Target & Mode & Dose & Code & Origin & Site & & & \\
\hline & MV & $2 \mathrm{~Gy}$ & HT1299 & Lung ca & intravenous & $6 a$ & CXCR4 & (Gu et al., 2015a) \\
\hline \multicolumn{9}{|c|}{ Inoculation site } \\
\hline & $220 \mathrm{kV}$ & 20 Gy & R18 & Melanoma & intradermal & $2^{b}$ & $\mathrm{O}_{2} / \mathrm{UPAR}$ & (Rofstad et al., 2005) \\
\hline & $220 \mathrm{kV}$ & $20 \mathrm{~Gy}$ & D12 & Melanoma & intradermal & 2 & $\mathrm{O}_{2} / \mathrm{LL} 8$ & (Rofstad et al., 2005) \\
\hline & $220 \mathrm{kV}$ & 20 Gy & SCCVII & Oral ca & subcutaneous & 3.5 & CYR61; av $\beta 5$ & (Monnier et al., 2008) \\
\hline & $220 \mathrm{kV}$ & $20 \mathrm{~Gy}$ & НCT116 & Colon ca & subcutaneous & 4 & & \\
\hline \multicolumn{9}{|c|}{ Total body } \\
\hline & $6 \mathrm{MV}$ & $0.3 \mathrm{~Gy}$ & $4 \mathrm{~T} 1$ & mammary ca & mammary f.p. & 7 & VEGFR & (Sofia Vala et al., 2010) \\
\hline & $6 \mathrm{MV}$ & $0.3 \mathrm{~Gy}$ & MOLT4 & leukemia & intravenous & 5 & & \\
\hline \multicolumn{9}{|l|}{ Tumor } \\
\hline & $220 \mathrm{kV}$ & $10 \mathrm{~Gy}$ & R18 & Melanoma & intradermal & $1.6^{\mathrm{b}}$ & $\mathrm{O}_{2} /$ UPAR & (Rofstad et al., 2004) \\
\hline & $250 \mathrm{kV}$ & $2 \times 4 \mathrm{~Gy}$ & U251 & glioma & intracranial & $>1^{d}$ & MMP2 & (Badiga et al., 2011) \\
\hline & ${ }^{60} \mathrm{Co}$ & $5 \times 10$ Gy & C6L & glioma & subcutaneous & $>1^{a}$ & EMT & (Park et al., 2012) \\
\hline & $\mathrm{nm}$ & $5 \times 2 \mathrm{~Gy}$ & FCT133 & Thyroid ca & subcutaneous & 1.6 & PI3K & (Burrows et al., 2013) \\
\hline & $\mathrm{nm}$ & $20 \mathrm{~Gy}$ & $4 \mathrm{~T} 1$ & mammary ca & mammary f.p. & $5^{e}$ & & (Vilalta et al., 2014) \\
\hline & $6 \mathrm{MeV}$ & 2 Gy & SW480 & Colon ca & intracolonic & $>1^{a}$ & & (Timaner et al., 2015) \\
\hline & $X$-rays & $15 \times 2 G y$ & SGC7901 & Gastric ca & subcutaneous & 2 & & (Zhang et al., 2015a) \\
\hline
\end{tabular}

Scores indicate the metastasis in the lungs unless indicated as: ${ }^{a}$, lung and other metastases; ${ }^{b}$, lymph node metastasis; ${ }^{c}$, circulating tumor cells (CTC); ${ }^{d}$, Local invasion; ', self-seeding from an unirradiated heterolateral breast tumor to an irradiated recipient one. Scores represent metastasis after administration of radiation relative to control mice (Ir/Ctrl). Abbreviations; avb3, integrin; CAF, cancer-associated fibroblasts; C-ion, carbon-ions; Ca, carcinoma; CAF, cancerassociated fibroblasts; CCL2, C-C motif chemokine ligand 2; CM, conditioned medium; Co, cobalt; COX-2, cyclooxygenase-2; Cs, cesium; CTC, circulating tumor cells; CXCL12, C-X-C motif chemokine 12; CXCR4, C-X-C chemokine receptor type 4; CYR61, Cysteine-rich angiogenic inducer 61; EMT, epithelial-to-mesenchymal transition; f.p., fat pad; Gy, gray; HIF, hypoxia-inducible factor; IGFR, insulin-like growth factor receptor; IL, interleukin; KITL, tyrosine-kinase receptor encoded by the KIT locus, also known as stem cell factor; Met, tyrosine-protein kinase Met; MMP, matrix metalloproteinases; nm, not mentioned; PI3K, phosphatidylinositol 3-kinase; Ras, small GTPase encoded by the RAS gene; sa, sarcoma; TGF-b, transforming growth factor beta; uPAR, urokinase-type plasminogen activator receptor; VEGFR, vascular endothelial growth receptor

such as TIMPs. Proteases not only degrade ECM but also cleave off growth factors from their precursors, e.g. proteases activate TGF- $\beta$ through cleavage from its inactive precursor latency-associated peptide (LAP- $\beta 1$ ). Both enzymatic activities are enhanced by ionizing radiation, explaining stimulation of invasion and metastasis-related cellular activities (Tables 2 and 3). Irradiation enhanced activity of MMP9 is explained by enhanced transcription via NF-kB (Park et al., 2006a; Cheng et al., 2006a). Irradiationenhanced MMP2 promoter activity, mRNA transcription, and protein secretion associated with invasiveness was observed in glioma cells lacking functional PTEN (U87, U251, U373, and C6) but not in those harboring wild-type PTEN (LN18 and LN428) (Park et al., 2006a; Cordes et al., 2003). This is in contrast with the observations of WildBode et al. who reported upregulated expression of MMP2 and MT1-MMP, downregulated expression of TIMP-2, and increased invasiveness in glioma cells regardless of PTEN status (Wild-Bode et al., 2001). In LLC-LM murine lung cancer cells, irradiation prompted increased expression of mRNA and protein as well as increased enzyme activity for MMP9 but not for MMP2 (Chou et al., 2012). These researchers also observed that the administration of $5 \times 10$ Gy to the subcutaneous tumor in the thigh enhanced the MMP9 level, stimulated entry of cancer cells into the circulation and increased the number of pulmonary metastasis, whereas $2 \times 30$ Gy reduced these parameters. Implication of the $\beta$-catenin/TCF signaling pathway links MMP to the EMT phenotype. In U87 glioma cells, irradiation increased the $\beta$-catenin/ TCF transcriptional activity, followed by an upregulation of its downstream genes, MMP2, and MMP9 (Dong et al., 2015a). Interestingly, irradiation-induced overexpression of growth arrest and DNA damage inducible protein alpha (Gadd45a) inhibits the nuclear translocation of $\beta$-catenin, resulting in a downregulation of MMP9, an increase in E-cadherin and a delayed medulloblastoma tumor development (Asuthkar et al., 2011). Other hydrolases are also involved in irradiation-induced invasion, e.g. the activation of the serine protease uPA in pancreatic cancer cells via carbon-ion irradiation (Fujita et al., 2014a). 
As stated above, activation of TGF- $\beta$ through release from LAP- $\beta 1$ is an extracellular process, that occurs rapidly (within $24 \mathrm{~h}$ ), persistently (7 days) and dosedependently (from 0.1 Gy to 5 Gy) upon irradiation of the mouse mammary gland (Ehrhart et al., 1997). Such activation results from ROS-induced oxidation of LAP$\beta 1$ triggering a conformational change that releases TGF- $\beta 1$. A methionine residue at amino acid position 253 unique to LAP- $\beta 1$ is critical to this ROS-mediated activation (Jobling et al., 2006). Circulating levels of TGF- $\beta$ were increased in a mouse model of irradiationenhanced metastatic breast cancer, regardless of the site of irradiation: thorax, pelvis or primary tumor and before or after injection of the cancer cells. In this experiment, circulating cancer cells did not grow ex vivo in the presence of the TGF- $\beta$ antibody and irradiation failed to enhance lung metastases in mice bearing tumors that lacked the type II TGF- $\beta$ receptor, suggesting that the increase in metastases was due to a direct effect of TGF- $\beta$ on the cancer cells (Biswas et al., 2007).

Heterotypic cell-cell adhesion Heterotypic cell-cell adhesion, implicated in extravasation and metastasis, is facilitated by irradiation-triggered expression of cell adhesion factors such as E-selection and vascular cell adhesion protein (VCAM) on cancer cells and endothelial cells (Hamalukic et al., 2011). Also cell-substrate adhesion is enhanced by irradiation as quantified by the number of cells that resist washing after seeding and incubation on tissue culture plastic substrate coated with elements from the extracellular matrix (Tsutsumi et al., 2009; Nalla et al., 2010; Akino et al., 2009; Cordes et al., 2003; Jung et al., 2007; Ogata et al., 2005).

The type of extracellular matrix influenced clonogenic survival of some glioblastoma cells with irradiationenhancement on fibronectin or Matrigel, but not bovine serum albumin (Cordes et al., 2003). For irradiationenhanced cell-substrate adhesion, little or no difference was found between collagen 1, fibronectin, vitronectin or laminin (Akino et al., 2009; Ogata et al., 2005; Lee et al., 2010). Irradiation-enhanced cell-substrate adhesion and wound-healing migration could be obtained only when the tissue culture substrate was coated with collagen 1 (Tsutsumi et al., 2009).

The pro-invasive effect of fibroblasts on pancreatic cancer cells has been ascribed to hepatocyte growth factor (HGF) secreted by the fibroblasts and interacting with the tyrosine-protein kinase Met (c-MET) on the cancer cells by Ohuchida et al. (Ohuchida et al., 2004), and to the ubiquitous and polyfunctional chemokine CXCL12 interacting with CXCR4 on the cancer cells by Li et al. (Li et al., 2016a) HGF was put forward because of the activation of c-MET in pancreatic cancer cells exposed to irradiated fibroblasts and neutralization of this effect by NK4 (an antagonist of HGF), although increased release of HGF from the fibroblasts could not be demonstrated. CXCL12 was selected because it emerged as the most elevated species among the irradiation-enhanced levels of chemokine mRNA. To confirm, Li et al. showed that addition of CXCL12 to the co-culture stimulated invasion, whereas the CXCR4 partial agonist AMD3100 reduced it. CXCL12 together with macrophage migration inhibitory factor (MIF) were chosen also to explain stimulation of adhesion and trans-endothelial migration of human breast cancer cells by irradiation of lung cells on the basis of a high fold change found in a 52 cytokine secretome assay ( $\mathrm{Li}$ et al., 2016a). These pro-invasive cellular activities were largely mimicked by recombinant CXCL12 and MIF, whereas an allosteric inhibitor of the CXCR4 receptor prevented these activities (Feys et al., 2015).

In summary, irradiation might stimulate motility and invasion on various different levels. This effect seems to be dependent on dose, cell line and type of irradiation (photons versus heavy ions), and was sometimes only transient.

\section{Metastasis}

To analyze the mechanisms of metastasis, cancer cells were inoculated at various sites, orthotopic or paratopic, in immunosuppressed or syngeneic hosts (Table 3), producing models that are more readable than the natural situation at the risk of being less relevant. Similarly, manipulation of the target and the time of radiation facilitates dissection of mechanism at the price of lower relevance. In this way an increase in the metastatic potential has been observed upon radiation of the cancer cells or of the host, be it on the site of inoculation, putative sites of metastasis or whole body, prior to transfer of cancer cells. Also the intravenous inoculation of radio-resistant cancer cells that were sub-cultured for several passages after irradiation yielded an increased metastatic potential ( $\mathrm{Su}$ et al., 2012). The transfer of cancer cells into preirradiated host tissue is a model of recurrent primary tumors in humans. This model has proven the tumor bed effect, a phenomenon which was originally described as a prolonged latency period and a reduced volumetric growth rate of tumors implanted in previously irradiated tissue and was later extended to an increased metastatic potential of tumors in preirradiated beds (Stenstrom et al., 1955; Milas et al., 1988; Rofstad et al., 2005). For instance, irradiation of the intradermal inoculation site in nude mice caused enhanced lung metastasis from human melanoma tumors (Rofstad et al., 2005) and irradiation of the mammary fat pad of syngeneic mice $24 \mathrm{~h}$ before inoculation of D2A1 breast cancer cells enhanced invasion, CTCs and lung metastasis upon orthotopic but not upon intravenous inoculation (Bouchard et al., 2013). 
As for irradiation of putative sites of metastasis, contradictory results have been noted. On the one hand, Travis et al. observed that irradiation of the whole lung $(14,5$ or $20 \mathrm{~Gy})$ after the subcutaneous growth of sarcoma $\mathrm{F}$ tumors in CBA mice led to a decrease in lung metastases (Travis et al., 1981). On the other hand, several others have reported an increase in lung metastases after irradiation of the lungs prior to subcutaneous injection of TS/adenocarcinoma cells or B16 melanoma cells or prior to intravenous injection of B16 melanoma cells, TS/adenocarcinoma cells (Klein et al., 2016), fibrosarcoma cells (Withers \& Milas, 1973), mammary carcinoma cells (Dao \& Yogo, 1967), Ehrlich ascites cells (Tanaka, 1976) or Walker tumor cells (Fisher \& Fisher, 1969). Furthermore, Gong et al. reported that in BALB/c mice, irradiation of the right side of the chest to $9 \mathrm{~Gy}$ after injection of 4T1 mammary carcinoma tumor cells in the right last mammary gland led to higher incidence of lung metastasis and a shorter survival (Gong et al., 2015). Interestingly, Feys et al. observed enhanced lung metastasis from orthotopic 4T1mouse mammary tumors after partial right lung irradiation, but not after whole thorax irradiation (Feys et al., 2015). Also irradiation of the liver has been reported to lead to an increase in liver metastases after the intraportal inoculation of Ehrlich ascites carcinoma cells (Fisher \& Fisher, 1969; Koike et al., 1962). Several hypotheses of this pro-metastatic effect of radiotherapy on the putative site of metastasis have been formulated. (a) Circulating tumor cells might be attracted to the irradiated tissue. (b) Cancer cell release from the primary tumor might be stimulated. (c) Irradiation could instigate local immune changes leading to the recruitment of several immune cells from the innate and adaptive immune system, which can produce paracrine signals facilitating tumor survival and growth (Feys et al., 2015; Gong et al., 2015). These immune cells can however also stimulate an antitumor immune response, which is therefore not in line with the previously discussed observations. (d) Irradiation could damage vascular endothelial cells which enables the transmigration of tumor cells through the endothelium (Klein et al., 2016).

Via bioluminescence it was proven that irradiation of the primary breast tumor increases the homing of donor tumor cells to this site and therefore enhances self-seeding (Vilalta et al., 2014). Timaner et al. showed in mice that not only local irradiation of the tumor with 2 Gy will cause an increase in metastases, but also that irradiation ( 2 Gy) of the abdomen, prior to intravenous injection of CT26 colon cancer cells into the tail vein leads to higher mortality due to pulmonary metastases (Timaner et al., 2015). This finding indicates that irradiation can instigate a systemic host response that enhances migration and invasion of tumor cells.
Delivery of an ablative dose to the primary tumor and a subsequent increase in lung metastases as compared to no irradiation of the primary tumor led Camphausen et al. to conclude that metastases are present in a dormant form at the moment of irradiation, similar to the experiments in which the same type of tumors were removed surgically (Camphausen et al., 2001; O'Reilly et al., 1994). With a subtherapeutic dose, irradiationenhanced metastasis was observed only after regrowth of the primary tumor, leading to the conclusions that irradiation stimulated metastasis from the primary tumor (Rofstad et al., 2005).

An overview of the communication within the cancer ecosystem regarding invasion and metastasis is depicted in Fig. 2. The molecular pathways implicated in the process of radiation-enhanced invasion and metastasis is depicted in Fig. 1. There is large and convincing preclinical evidence in favor of a prometastatic activity of radiation with very few scores lower than zero (seventh column in Table 3), pointing to inhibition. Furthermore, in vitro and in vivo experiments have revealed a profusion of molecular pathways implicated in radiationenhanced invasion and metastasis (Fig. 1), several of which are sensitive to inhibition by molecules that are clinically applicable (Table 2). The crucial question is: how do these experimental findings translate to the clinical situation?

\section{Clinical evidence}

Preclinical evidence on how irradiation influences the metastatic process is conflicting, and in vitro and in vivo experiments are often not a good representation of the human situation. Furthermore, the potential pro-metastatic effect of radiotherapy is hard to infer from the literature due to lack of attention to metastasis in palliative cases, heterogeneity of the cohorts analyzed, lower sensitivity or specificity of methods of detection, lack of systematic autopsy and weak study designs. Nonetheless, clinical evidence exists that certain cancer therapies produce prometastatic and pro-invasive signals.

Chung et al. analyzed a cohort of 340 patients with unresectable hepatocellular carcinoma (T3NOM0) who received either transcatheter arterial chemoembolization alone or in combination with radiotherapy (40-66 Gy in 1.8 to 2 Gy fractions). Although the study was not randomized, baseline patient characteristics and treatment variables did not differ significantly between both groups. Overall survival was similar in both groups, yet the group treated with radiotherapy quickly developed intra- and extrahepatic tumors outside the irradiation field resulting in a significantly shorter extrahepatic progressionfree survival (Chung et al., 2006). The smaller study by Cheng et al. had similar findings (Chia-Hsien Cheng et al., 2001). A mechanism postulated is the irradiation-induced 


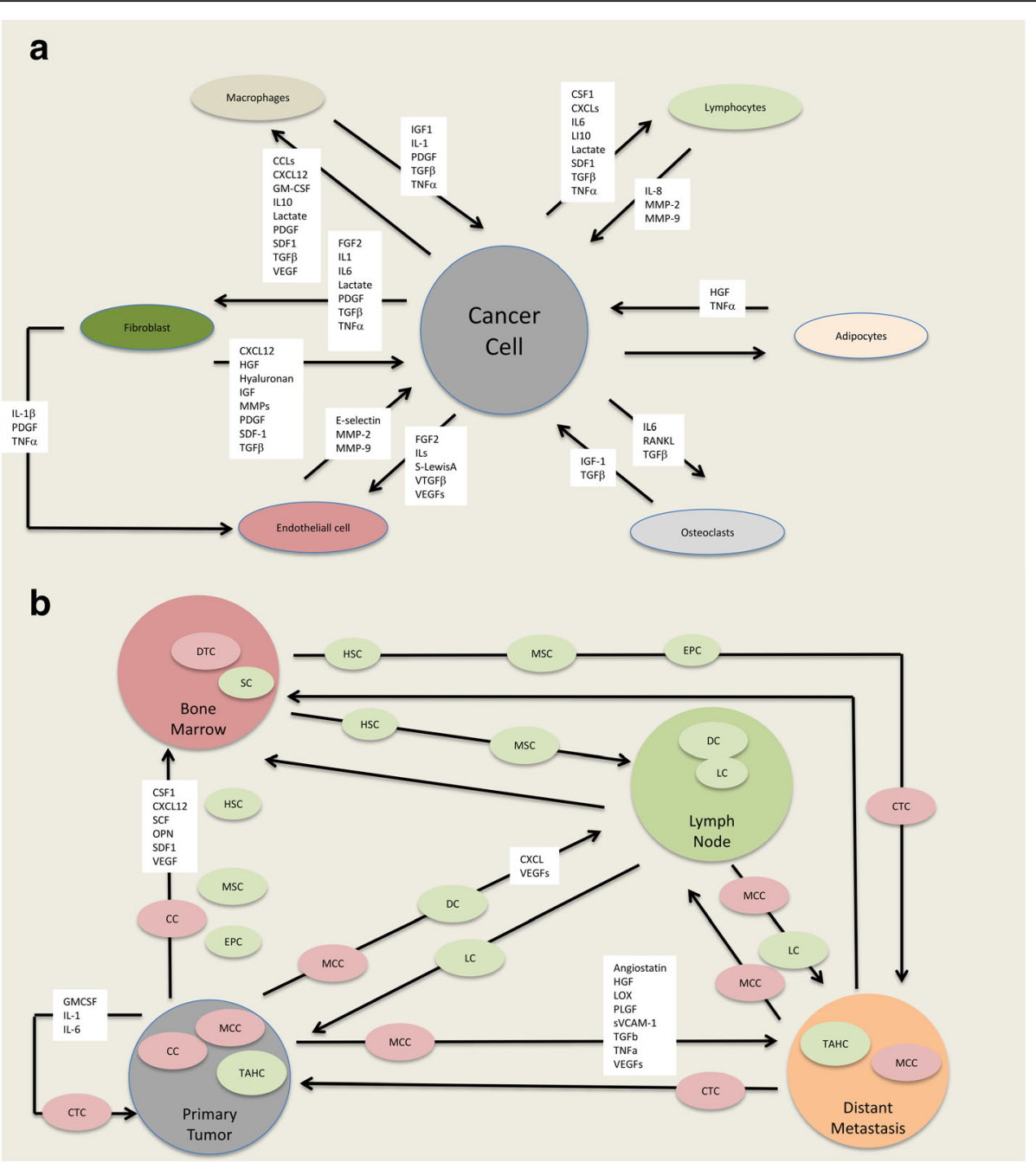

Fig. 2 Schematics of mutual communication between cancer cells, tumor associated host cells and ecosystems. a Schematic of communication between cancer cells and tumor-associated host cells with invasion-related radiation-sensitive molecules. Arrows indicate the communication between cancer cells and tumor-associated host cells, namely fibroblasts, macrophages, lymphocytes, adipocytes, osteoclasts and endothelial cells, and between tumor-associated host cells, for example fibroblasts to endothelial cells. White text boxes overlapping the arrows contain the molecules implicated in this communication and shown to be sensitive to alterations by radiation. This intercellular communication establishes the individual local ecosystems that participate at metastasis as shown in panel b Adapted from Mareel et al., (2009b), with data from: Vakaet (2004); Abdollahi (2005); Chargari et al., (2013); De Bacco et al., (2011b); Gu et al., (2015b); Hamalukic et al., (2011b); Hirschhaeuser et al., (2011); Kuonen et al., (2012a); Li et al., (2016b); Madani et al., (2008); Nubel (2004); Vilalta (2016); Lee et al., (2017c). b Schematic of the communication between ecosystems of primary tumor, distant metastasis, lymph node and bone marrow, associated with metastasis and sensitive to ionizing radiation. Arrows indicate the communication between ecosystems, namely the primary tumor, lymph node, distant metastasis and bone marrow. Participating at this communication are host cells (smaller green ovals), cancer cells (smaller pink ovals) and molecules (white text boxes), all implicated in radiation-enhanced metastasis. Adapted from: Madani et al., (2008); Ceelen et al., (2014) and Willaert et al., (2014) with data from: Kioi et al., (2010b); Kuonen et al., (2012c); Russell \& Brown (2013b); Vilalta (2016). Abbreviations: CC, cancer cells; CCL, C-C motif chemokine ligand; CSF1, macrophage colony-stimulating factor 1; CTC, circulating tumor cells; CXCL, C-X-C motif chemokine ligand; DC, dendritic cells; DTC,disseminated tumor cells; EPC, endothelial precursor cells; FGF2, basic fibroblast growth factor; GMCSF, granulocyte-macrophage colony-stimulating factor; HGF, hepatocyte growth factor; HSC, hematopoietic stem cells; IGF1, insulinlike growth factor 1; LL, interleukin; LC, lymphocytes; MCC, metastatic cancer cells; MMP, matrix metalloproteinase; MSC, mesenchymal stem cells; OPN, osteoprotogerin; PDGF, platelet-derived growth factor; PIGF, placental growth factor; RANKL, receptor activator of nuclear kappa-B ligand; SC, stem cells; S-Lewis A, sialyl-Lewis A antigen; SCF, stem cell factor; SDF1 (also called CXCL12), stromal- cell derived factor 1; SVCAM1, soluble vascular cell adhesion molecule 1; TGF $\beta$, transforming growth factor beta; TNFa, tumor necrosis factor-alpha; VEGF, vascular endothelial growth factor

upregulation of VEGF and enhanced intra-tumor angiogenesis (Chung et al., 2006). In line with these findings, in 12 of 16 stage III rectal cancer patients, upregulation of VEGF was shown immunohistochemically on the resection specimen after neo-adjuvant radiochemotherapy (Nozue et al., 2001).

In a prospective randomized trial, patients with oroand hypopharyngeal squamous cell carcinoma were either 
treated with surgery or with radiotherapy (5 x 4Gy) followed 3 days later by surgery. No difference in survival was seen, yet the group treated with radiotherapy had a higher incidence of distant metastases at time of death (Strong et al., 1978). Other non-randomized studies in head and neck cancer reported more distant metastases in the radiotherapy groups; yet selection bias could be the cause of this difference (Merino et al., 1977; Schantz \& Peters, 1987).

These findings bring forth the hypothesis that the beneficial local effects of radiotherapy are counteracted by its systemic pro-invasive and pro-metastatic effect and therefore does not lead to an increased overall survival. The fact that the appearance of distant metastasis has been unchanged in the field of head and neck squamous cell carcinoma over the last decades, notwithstanding new radiotherapy and chemotherapy, supports this hypothesis (Duprez et al., 2017). The addition of molecules blocking this systemic pro-invasive and prometastatic effect could therefore entail a survival benefit, examples of these molecules are denoted in Table 2. The combination of radiotherapy with targeted molecules has been previously reviewed by Maier et al. (Maier et al., 2016).

Furthermore, it has been shown that radiotherapy and chemotherapy can alter the metastatic pattern as compared to no treatment. De La Monte et al. found that the distribution of metastases of small cell lung carcinoma was different after radiotherapy, chemotherapy or no therapy, but with similar number of total distant metastases and similar overall survival in all groups. These cancer treatments therefore might instigate a growth advantage to certain subpopulations of the tumor, which metastasize to specific sites (de la Monte et al., 1988).

Radiotherapy can also induce a local pro-invasive effect, as shown predominantly in breast cancer but also in nasopharyngeal and endometrial cancer, in which inflammatory skin recurrence, exactly demarcating the previously irradiated area, occurred (Tsujino et al., 2011; Meltzer et al., 1981; Marley \& Marley, 1982). This phenomenon is rare and its incidence in breast cancer is estimated at $0.25 \%$. The mechanism inducing it is not understood, yet radiotherapy could change the local microenvironment, facilitating tumor cells to migrate into this area (Tsujino et al., 2011).

In line with this, radiotherapy of prostate cancer and rectal cancer can increase MMP activity in the tumor and the local microenvironment (Angenete et al., 2009; Hovdenak et al., 2002; Kumar et al., 2002; Kumar et al., 2000). As stated above, these MMPs play a crucial role in invasion (Brown \& Murray, 2015) and are correlated with cancer stage, metastasis and survival (Cho et al., 2005; Trudel et al., 2010).
Evidently, radiotherapy can also yield an anti-invasive and anti-metastatic effect. Post-mastectomy radiotherapy in high-risk breast cancer patients leads to a significantly lower probability of locoregional recurrences and distant metastases (Nielsen et al., 2006; Poortmans et al., 2015). Furthermore, several pre-clinical and clinical data indicate that radiotherapy can instigate a systemic antitumor effect in non-irradiated metastases, yet this effect is extremely rare and impossible to predict (Abuodeh et al., 2016; Formenti \& Demaria, 2009). The underlying mechanism could be a radiotherapy-induced systemic anti-tumor immune response (Formenti \& Demaria, 2009). The systemic anti-tumor effect of radiotherapy was described for the first time by Mole in 1953 and was termed the 'abscopal effect' (Mole, 1953). Recently, it has been more frequently reported, often when radiotherapy was administered in combination with checkpointinhibitors (Postow et al., 2012). Small prospective studies and retrospective studies have shown that the combination of checkpoint-inhibitors with radiotherapy could act synergistically, raising response rates and improving survival (Shaverdian et al., 2017; Twyman-Saint Victor et al., 2015; Hiniker et al., 2016; Williams et al., 2017; Tang et al., 2017; Sundahl et al., 2018). The underlying molecular mechanism could be the release of tumor antigen, triggered by radiotherapy-induced immunogenic cell death, and a subsequent systemic anti-tumor immune response, mediated by the checkpoint-inhibitor which reactivates the immune system by blocking the inhibitory signals on the immune cells (e.g. programmed cell death protein 1) (Formenti \& Demaria, 2009). Several prospective clinical trials are currently investigating this combination treatment (Vacchelli et al., 2016; Sundahl et al., 2017; De Wolf et al., 2017).

\section{Conclusion}

There is abundant evidence from in vivo and in vitro experiments that ionizing radiation, applied to cancer cells or host cells, to the tumor, the whole host or the putative site of tumor development may stimulate the metastatic process. Clinical observations do not directly confirm these data, leaving open the question whether or not radiotherapy enhances metastasis. The overall net outcome of radiotherapy is beneficial as it may reduce metastasis and prolong survival. This therapeutic benefit does not exclude a limitation of the benefit by negative effects of the irradiation on the cancer cells or on the host. The present review of literature suggests clinical trials to investigate correction of the pro-invasive and pro-metastatic activities of radiation by anti-inflammatory or other agents shown to be effective in preclinical settings.

\section{Abbreviations}

BCl-X $\mathrm{X}_{\mathrm{L}}$ B cell lymphoma-extra large; CAM: Chorioallontoic membrane; CCL: Chemokine ligand; C-MET: Tyrosine-protein kinase Met; CTC: Circulating tumor cells; CTLA4: Cytotoxic T-lymphocyte-associated protein 4; 
CYR61: Cysteine-rich angiogenic inducer 61; DTC: Disseminated tumor cells; EGFR: Epidermal growth factor receptor; EMT: Epithelial mesenchymal transition; FAK: Focal adhesion kinase; Gadd45a: Growth arrest and DNA damage inducible protein alpha; G-CSF: Granulocyte-colony stimulating

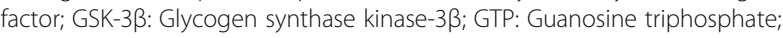
Gy: Gray; HGF: Hepatocyte growth factor; HIF: Hypoxia inducible factor; HUVEC: Human umbilical vein endothelial cells; ILK: Integrin-linked kinase; IKB: Inhibitor of nuclear factor of kappa-light-chain-enhancer of activated B cell; LAP- $\beta 1$ : Latency-associated peptide of TGF $\beta$; MIF: Migration inhibitory factor; MMP: Matrix metalloproteinase; MSC: Mesenchymal stromal cell; MT1MMP: Membrane-bound matrix metalloproteinase-1; PI3K: Phosphoinositide 3-kinase; PINCH1: Particularly interesting new cysteine-histidine-rich 1; PTEN: Phosphatase and tensin homolog; ROS: Reactive oxygen species; Snail: Zinc finger protein SNAI1; STAT3: Signal transducer and activator of transcription factor 3; TBI: Total body irradiation; TBK-1: Tank-binding kinase-1; TCF: T-cell factor; TIMP: Tissue inhibitors of matrix metalloproteinase; Trex1: Three prime repair exonuclease 1; UPA: Urokinase-type plasminogen activator; VCAM: Vascular cell adhesion molecule; VEGF: Vascular endothelia growth factor; ZEB1: Zinc finger E-box-binding homeobox 1

\section{Acknowledgements}

Not applicable.

\section{Funding}

No funding was received for writing this manuscript.

\section{Availability of data and materials}

Data sharing is not applicable to this article as no datasets were generated or analyzed during the current study.

\section{Authors' contributions}

NS and MM drafted the main manuscript. FD, PO and WDN gave valuable comments and insights. All authors read and approved the final manuscript.

\section{Ethics approval and consent to participate}

Not applicable.

\section{Consent for publication}

Not applicable.

\section{Competing interests}

The authors declare that they have no competing interests.

\section{Publisher's Note}

Springer Nature remains neutral with regard to jurisdictional claims in published maps and institutional affiliations.

Received: 11 January 2018 Accepted: 15 March 2018

Published online: 24 April 2018

\section{References}

Abdollahi A. Inhibition of Â vÂ 3 Integrin Survival Signaling Enhances Antiangiogenic and Antitumor Effects of Radiotherapy. Clinical Cancer Research. 2005;11(17):6270-9.

Abdollahi A, Griggs DW, Zieher H, Roth A, Lipson KE, Saffrich R, Grone HJ, Hallahan DE, Reisfeld RA, Debus J, et al. Inhibition of alpha(v)beta3 integrin survival signaling enhances antiangiogenic and antitumor effects of radiotherapy. Clin Cancer Res. 2005;11:6270-9.

Abuodeh Y, Venkat P, Kim S. Systematic review of case reports on the abscopal effect. Curr Probl Cancer. 2016;40:25-37.

Ahmed KM, Zhang H, Park CC. NF-kappaB regulates radioresistance mediated by beta1-integrin in three-dimensional culture of breast cancer cells. Cancer Res. 2013a;73:3737-48.

Ahmed KM, Zhang H, Park CC. NF-Â B Regulates Radioresistance Mediated By Â 1-Integrin in Three-Dimensional Culture of Breast Cancer Cells. Cancer Research. 2013b;73(12):3737-48.

Akino Y, Teshima T, Kihara A, Kodera-Suzumoto Y, Inaoka M, Higashiyama S, Furusawa Y, Matsuura N. Carbon-ion beam irradiation effectively suppresses migration and invasion of human non-small-cell lung cancer cells. Int J Radiat Oncol Biol Phys. 2009;75:475-81.
Albert JM, Cao C, Geng L, Leavitt L, Hallahan DE, Lu B. Integrin alpha v beta 3 antagonist Cilengitide enhances efficacy of radiotherapy in endothelial cell and non-small-cell lung cancer models. Int J Radiat Oncol Biol Phys. 2006:65:1536-43.

Angenete E, Oresland T, Falk P, Breimer M, Hultborn R, Ivarsson ML. Preoperative radiotherapy and extracellular matrix remodeling in rectal mucosa and tumour matrix metalloproteinases and plasminogen components. Acta Oncol. 2009:48:1144-51.

Asuthkar S, Nalla AK, Gondi CS, Dinh DH, Gujrati M, Mohanam S, Rao JS. Gadd45a sensitizes medulloblastoma cells to irradiation and suppresses MMP-9mediated EMT. Neuro-Oncology. 2011;13:1059-73.

Badiga AV, Chetty C, Kesanakurti D, Are D, Gujrati M, Klopfenstein JD, Dinh DH, Rao JS. MMP-2 siRNA inhibits radiation-enhanced invasiveness in glioma cells. PLoS One. 2011;6:e20614.

Baker D, Elkon D, Lim ML, Constable W, Wanebo H. Dose local x-irradiation of a tumor increase the incidence of metastases? Cancer. 1981;48:2394-8.

Barker HE, Paget JT, Khan AA, Harrington KJ. The tumour microenvironment after radiotherapy: mechanisms of resistance and recurrence. Nat Rev Cancer. 2015;15:409-25.

Bastos LG, de Marcondes PG, de-Freitas-Junior JC, Leve F, Mencalha AL, de Souza WF, de Araujo WM, Tanaka MN, Abdelhay ES, Morgado-Diaz JA. Progeny from irradiated colorectal cancer cells acquire an EMT-like phenotype and activate Wnt/beta-catenin pathway. J Cell Biochem. 2014;115:2175-87.

Biswas S, Guix M, Rinehart C, Dugger TC, Chytil A, Moses HL, Freeman ML, Arteaga CL. Inhibition of TGF-beta with neutralizing antibodies prevents radiation-induced acceleration of metastatic cancer progression. J Clin Invest. 2007;117:1305-13.

Bouchard G, Bouvette G, Therriault H, Bujold R, Saucier C, Paquette B. Preirradiation of mouse mammary gland stimulates cancer cell migration and development of lung metastases. Br J Cancer. 2013;109:1829-38.

Brown GT, Murray Gl. Current mechanistic insights into the roles of matrix metalloproteinases in tumour invasion and metastasis. J Pathol. 2015;237: 273-81.

Burrows N, Telfer B, Brabant G, Williams KJ. Inhibiting the phosphatidylinositide 3kinase pathway blocks radiation-induced metastasis associated with rhoGTPase and hypoxia-inducible factor-1 activity. Radiother Oncol. 2013;108: 548-53.

Camphausen K, Moses MA, Beecken WD, Khan MK, Folkman J, O'Reilly MS. Radiation therapy to a primary tumor accelerates metastatic growth in mice. Cancer Res. 2001;61:2207-11.

Ceelen W, Smeets P, Backes W, Van Damme N, Boterberg T, Demetter P, Bouckenooghe I, De Visschere M, Peeters M, Pattyn P. Noninvasive monitoring of radiotherapy-induced microvascular changes using dynamic contrast enhanced magnetic resonance imaging (DCE-MRI) in a colorectal tumor model. Int J Radiat Oncol Biol Phys. 2006;64:1188-96.

Ceelen W, Pattyn P, Mareel M. Surgery, wound healing, and metastasis: Recent insights and clinical implications. Critical Reviews in Oncology/Hematology. 2014;89(1):16-26.

Chang L, Graham PH, Hao J, Ni J, Bucci J, Cozzi PJ, Kearsley JH, Li Y. Acquisition of epithelial-mesenchymal transition and cancer stem cell phenotypes is associated with activation of the PI3K/Akt/mTOR pathway in prostate cancer radioresistance. Cell Death Dis. 2013a;4:e875.

Chang L, Graham PH, Hao J, Ni J, Bucci J, Cozzi PJ, Kearsley JH, Li Y. Acquisition of epithelial-mesenchymal transition and cancer stem cell phenotypes is associated with activation of the PI3K/Akt/mTOR pathway in prostate cancer radioresistance. Cell Death and Disease. 2013b;4:e875.

Chargari C, Clemenson C, Martins I, Perfettini J-L, Deutsch E. Understanding the functions of tumor stroma in resistance to ionizing radiation: Emerging targets for pharmacological modulation. Drug Resistance Updates. 2013; 16(1-2):10-21.

Cheng JC, Chou CH, Kuo ML, Hsieh CY. Radiation-enhanced hepatocellular carcinoma cell invasion with MMP-9 expression through PI3K/Akt/NF-kappaB signal transduction pathway. Oncogene. 2006a;25:7009-18.

Cheng JC-H, Chou CH, Kuo ML, Hsieh C-Y. Radiation-enhanced hepatocellular carcinoma cell invasion with MMP-9 expression through PI3K/Akt/NF-IoB signal transduction pathway. Oncogene. 2006b;25(53):7009-18.

Chia-Hsien Cheng J, Chuang VP, Cheng SH, Lin YM, Cheng TI, Yang PS, Jian JJ, You DL, Horng CF, Huang AT. Unresectable hepatocellular carcinoma treated with radiotherapy and/or chemoembolization. Int J Cancer. 2001;96:243-52.

Cho JH, Hong WG, Jung YJ, Lee J, Lee E, Hwang SG, Um HD, Park JK. Gammaionizing radiation-induced activation of the EGFR-p38/ERK-STAT3/CREB-1-EMT 
pathway promotes the migration/invasion of non-small cell lung cancer cells and is inhibited by podophyllotoxin acetate. Tumour Biol. 2016a;37:7315-25.

Cho JH, Hong WG, Jung YJ, Lee J, Lee E, Hwang SG, Um HD, Park JK. Gammalonizing radiation-induced activation of the EGFR-p38/ERK-STAT3/CREB-1-EMT pathway promotes the migration/invasion of non-small cell lung cancer cells and is inhibited by podophyllotoxin acetate.Tumor Biol. 2016b;37(6):7315-25.

Cho YR, Kwon HC, Suh SH, Lee JH, Kim SH, Choi HJ, Lee HS, Roh MS, Hwang TH, Kim JS, Kim HJ. Expressions of matrix metalloproteinase-7 and -9 and their prognostic significances in rectal cancer. Cancer Res Treat. 2005;37:354-9.

Chou CH, Teng CM, Tzen KY, Chang YC, Chen JH, Cheng JC. MMP-9 from sublethally irradiated tumor promotes Lewis lung carcinoma cell invasiveness and pulmonary metastasis. Oncogene. 2012;31:458-68.

Chung YL, Jian JJ, Cheng SH, Tsai SY, Chuang VP, Soong T, Lin YM, Horng CF. Sublethal irradiation induces vascular endothelial growth factor and promotes growth of hepatoma cells: implications for radiotherapy of hepatocellular carcinoma. Clin Cancer Res. 2006;12:2706-15.

Cordes N, Hansmeier B, Beinke C, Meineke V, van Beuningen D. Irradiation differentially affects substratum-dependent survival, adhesion, and invasion of glioblastoma cell lines. Br J Cancer. 2003;89:2122-32.

Cui YH, Suh Y, Lee HJ, Yoo KC, Uddin N, Jeong YJ, Lee JS, Hwang SG, Nam SY, Kim MJ, Lee SJ. Radiation promotes invasiveness of non-small-cell lung cancer cells through granulocyte-colony-stimulating factor. Oncogene. 2015a; 34:5372-82.

Cui Y-H, Suh Y, Lee H-J, Yoo K-C, Uddin N, Jeong Y-J, Lee J-S, Hwang S-G, Nam S-Y, Kim M-J, Lee S-J. Radiation promotes invasiveness of non-small-cell lung cancer cells through granulocyte-colony-stimulating factor. Oncogene. 2015b;34(42): 5372-82.

Dao $\mathrm{TL}$, Yogo $\mathrm{H}$. Enhancement of pulmonary metastases by $\mathrm{x}$-irradiation in rats bearing mammary cancer. Cancer. 1967;20:2020-5.

De Bacco F, Luraghi P, Medico E, Reato G, Girolami F, Perera T, Gabriele P, Comoglio PM, Boccaccio C. Induction of MET by ionizing radiation and its role in radioresistance and invasive growth of cancer. J Natl Cancer Inst. 2011a;103:645-61

De Bacco F, Luraghi P, Medico E, Reato G, Girolami F, Perera T, Gabriele P, Comoglio PM, Boccaccio C. Induction of MET by lonizing Radiation and Its Role in Radioresistance and Invasive Growth of Cancer. JNCl: Journal of the National Cancer Institute. 2011b;103(8):645-61.

de la Monte SM, Hutchins GM, Moore GW. Altered metastatic behavior of small cell carcinoma of the lung after chemotherapy and radiation. Cancer. 1988; 61:2176-82.

de Marcondes PG, Andrés Morgado-Díaz J. The Role of EphA4 Signaling in Radiation-Induced EMT-Like Phenotype in Colorectal Cancer Cells. Journal of Cellular Biochemistry. 2017;118(3):442-5.

De Wolf K, Vermaelen K, De Meerleer G, Lambrecht BN, Ost P. The potential of radiotherapy to enhance the efficacy of renal cell carcinoma therapy. Oncoimmunology. 2015;4:e1042198.

De Wolf K, Kruse V, Sundahl N, van Gele M, Chevolet I, Speeckaert R, Brochez L, Ost P. A phase II trial of stereotactic body radiotherapy with concurrent antiPD1 treatment in metastatic melanoma: evaluation of clinical and immunologic response. Journal of Translational Medicine. 2017;12(1):157.

Desgrosellier JS, Cheresh DA. Integrins in cancer: biological implications and therapeutic opportunities. Nat Rev Cancer. 2010;10:9-22.

Dong Z, Zhou L, Han N, Zhang M, Lyu X. Wnt/beta-catenin pathway involvement in ionizing radiation-induced invasion of U87 glioblastoma cells. Strahlenther Onkol. 2015a;191:672-80.

Dong Z, Zhou L, Han N, Zhang M, Lyu X. Wnt/ß-catenin pathway involvement in ionizing radiation-induced invasion of U87 glioblastoma cells. Strahlenther Onkol. 2015b;191(8):672-80.

Duprez F, Berwouts D, De Neve W, Bonte K, Boterberg T, Deron P, Huvenne W, Rottey S, Mareel M. Distant metastases in head and neck cancer. Head Neck. 2017:39:1733-43.

Ehrhart EJ, Segarini P, Tsang ML, Carroll AG, Barcellos-Hoff MH. Latent transforming growth factor beta1 activation in situ: quantitative and functional evidence after low-dose gamma-irradiation. FASEB J. 1997;11:991-1002.

Eke I, Cordes N. Focal adhesion signaling and therapy resistance in cancer. Semin Cancer Biol. 2015a;31:65-75.

Eke I, Cordes N. Focal adhesion signaling and therapy resistance in cancer. Seminars in Cancer Biology. 2015b;31:65-75.

Feys L, Descamps B, Vanhove C, Vral A, Veldeman L, Vermeulen S, De Wagter C, Bracke M, De Wever O. Radiation-induced lung damage promotes breast cancer lung-metastasis through CXCR4 signaling. Oncotarget. 2015;6:26615-32.
Fisher ER, Fisher B. Effects of x-irradiation on parameters of tumor growth, histology, and ultrastructure. Cancer. 1969;24:39-55.

Formenti SC, Demaria S. Systemic effects of local radiotherapy. Lancet Oncol. 2009;10:718-26.

Fujita M, Imadome K, Endo S, Shoji Y, Yamada S, Imai T. Nitric oxide increases the invasion of pancreatic cancer cells via activation of the PI3K-AKT and RhoA pathways after carbon ion irradiation. FEBS Lett. 2014a;588:3240-50.

Fujita M, Imadome K, Shoji Y, Isozaki T, Endo S, Yamada S, Imai T. Carbon-ion irradiation suppresses migration and invasiveness of human pancreatic carcinoma cells MIAPaCa-2 via Rac1 and RhoA degradation. Int J Radiat Oncol Biol Phys. 2015a;93:173-80.

Fujita M, Otsuka Y, Imadome K, Endo S, Yamada S, Imai T. Carbon-ion radiation enhances migration ability and invasiveness of the pancreatic cancer cell, PANC-1, in vitro. Cancer Sci. 2012;103:677-83.

Fujita M, Otsuka Y, Yamada S, Iwakawa M, Imai T. X-ray irradiation and rho-kinase inhibitor additively induce invasiveness of the cells of the pancreatic cancer line, MIAPaCa-2, which exhibits mesenchymal and amoeboid motility. Cancer Sci. 2011;102:792-8.

Fujita M, Yamada S, Imai T. Irradiation induces diverse changes in invasive potential in cancer cell lines. Semin Cancer Biol. 2015b;35:45-52.

Fujita M, Imadome K, Endo S, Shoji Y, Yamada S, Imai T. Nitric oxide increases the invasion of pancreatic cancer cells via activation of the PI3K-AKT and RhoA pathways after carbon ion irradiation. FEBS Letters. 2014b;588(17):3240-50.

Fujita M, Yamada S, Imai T. Irradiation induces diverse changes in invasive potential in cancer cell lines. Seminars in Cancer Biology. 2015c;35:45-52.

Furmanova-Hollenstein P, Broggini-Tenzer A, Eggel M, Millard AL, Pruschy M. The microtubule stabilizer patupilone counteracts ionizing radiation-induced matrix metalloproteinase activity and tumor cell invasion. Radiat Oncol. 2013;8:105

Galarza TE, Mohamad NA, Taquez Delgado MA, Vedoya GM, Crescenti EJ, Bergoc RM, Martin GA, Cricco GP. Histamine prevents radiation-induced mesenchymal changes in breast cancer cells. Pharmacol Res. 2016;111:731-9.

Ghosh S, Kumar A, Tripathi RP, Chandna S. Connexin-43 regulates p38-mediated cell migration and invasion induced selectively in tumour cells by low doses of gamma-radiation in an ERK-1/2-independent manner. Carcinogenesis. 2014:35:383-95.

Gogineni VR, Nalla AK, Gupta R, Gujrati M, Klopfenstein JD, Mohanam S, Rao JS. alpha3beta1 integrin promotes radiation-induced migration of meningioma cells. Int J Oncol. 2011;38:1615-24.

Gong HY, Hu WG, Hu QY, Li XP, Song QB. Radiation-induced pulmonary injury accelerated pulmonary metastasis in a mouse model of breast cancer. Oncol Lett. 2015;10:3613-8.

Gu Q, He Y, Ji J, Yao Y, Shen W, Luo J, Zhu W, Cao H, Geng Y, Xu J, et al. Hypoxia-inducible factor 1alpha (HIF-1alpha) and reactive oxygen species (ROS) mediates radiation-induced invasiveness through the SDF-1alpha/ CXCR4 pathway in non-small cell lung carcinoma cells. Oncotarget. 2015a;6: 10893-907.

Gu Q, He Y, Ji J, Yao Y, Shen W, Luo J, Zhu W, Cao H, Geng Y, Xu J, Zhang S, Cao J, Ding W-Q. Hypoxia-inducible factor $1 a(H I F-1 a)$ and reactive oxygen species (ROS) mediates radiation-induced invasiveness through the SDF-1a/ CXCR4 pathway in non-small cell lung carcinoma cells. Oncotarget. 2015b;6(13),

Hamalukic M, Huelsenbeck J, Schad A, Wirtz S, Kaina B, Fritz G. Rac1regulated endothelial radiation response stimulates extravasation and metastasis that can be blocked by HMG-CoA reductase inhibitors. PLoS One. $2011 \mathrm{a} ; 6: \mathrm{e} 26413$.

Hamalukic M, Huelsenbeck J, Schad A, Wirtz S, Kaina B, Fritz G, Anderson KI. Rac1Regulated Endothelial Radiation Response Stimulates Extravasation and Metastasis That Can Be Blocked by HMG-CoA Reductase Inhibitors. PLoS ONE. 2011b;6(10):e26413.

He E, Pan F, Li G, Li J. Fractionated ionizing radiation promotes epithelialmesenchymal transition in human esophageal Cancer cells through PTEN deficiency-mediated Akt activation. PLoS One. 2015a;10:e0126149.

He E, Pan F, Li G, Li J. Fractionated lonizing Radiation Promotes EpithelialMesenchymal Transition in Human Esophageal Cancer Cells through PTEN Deficiency-Mediated Akt Activation. PLOS ONE. 2015b;10(5):e0126149.

Heisel MA, Laug WE, Stowe SM, Jones PA. Effects of X-irradiation on artificial blood vessel wall degradation by invasive tumor cells. Cancer Res. 1984;44:2441-5.

Hellevik T, Pettersen I, Berg V, Bruun J, Bartnes K, Busund LT, Chalmers A, Bremnes R, Martinez-Zubiaurre I. Changes in the secretory profile of NSCLCassociated fibroblasts after ablative radiotherapy: potential impact on angiogenesis and tumor growth. Transl Oncol. 2013;6:66-74. 
Hiniker SM, Reddy SA, Maecker HT, Subrahmanyam PB, Rosenberg-Hasson Y, Swetter SM, Saha S, Shura L, Knox SJ. A prospective clinical trial combining radiation therapy with systemic immunotherapy in metastatic melanoma. Int J Radiat Oncol Biol Phys. 2016;96:578-88.

Hirschhaeuser F, Sattler UGA, Mueller-Klieser W. Lactate: A Metabolic Key Player in Cancer. Cancer Research. 2011;71(22):6921-5.

Ho JN, Kang GY, Lee SS, Kim J, Bae IH, Hwang SG, Um HD. BCl-XL and STAT3 mediate malignant actions of gamma-irradiation in lung cancer cells. Cancer Sci. 2010a;101:1417-23.

Ho JN, Kang Y, Lee SS, Kim J, Bae IH, Hwang SG, Um HD. Bcl-XI and STAT3 mediate malignant actions of gamma-irradiation in lung cancer cells. Cancer Sci. 2010b;101(6):1417-23.

Hosseini H, Obradovic MM, Hoffmann M, Harper KL, Sosa MS, Werner-Klein M, Nanduri LK, Werno C, Ehrl C, Maneck M, et al. Early dissemination seeds metastasis in breast cancer. Nature. 2016:540:552-8.

Hovdenak N, Wang J, Sung CC, Kelly T, Fajardo LF, Hauer-Jensen M. Clinical significance of increased gelatinolytic activity in the rectal mucosa during external beam radiation therapy of prostate cancer. Int J Radiat Oncol Biol Phys. 2002:53:919-27.

Imaizumi N, Monnier Y, Hegi M, Mirimanoff RO, Ruegg C. Radiotherapy suppresses angiogenesis in mice through TGF-betaRI/ALK5-dependent inhibition of endothelial cell sprouting. PLoS One. 2010;5:e11084.

Jobling MF, Mott JD, Finnegan MT, Jurukovski V, Erickson AC, Walian PJ, Taylor SE, Ledbetter S, Lawrence CM, Rifkin DB, Barcellos-Hoff MH. Isoform-specific activation of latent transforming growth factor beta (LTGF-beta) by reactive oxygen species. Radiat Res. 2006;166:839-48.

Jung JW, Hwang SY, Hwang JS, Oh ES, Park S, Han IO. lonising radiation induces changes associated with epithelial-mesenchymal transdifferentiation and increased cell motility of A549 lung epithelial cells. Eur J Cancer. 2007:43: 1214-24.

Kaliski A, Maggiorella L, Cengel KA, Mathe D, Rouffiac V, Opolon P, Lassau N, Bourhis J, Deutsch E. Angiogenesis and tumor growth inhibition by a matrix metalloproteinase inhibitor targeting radiation-induced invasion. Mol Cancer Ther. 2005;4:1717-28.

Kambach DM, Sodi VL, Lelkes PI, Azizkhan-Clifford J, Reginato MJ. ErbB2, FoxM1 and 14-3-3zeta prime breast cancer cells for invasion in response to ionizing radiation. Oncogene. 2014;33:589-98.

Kaminski MJ, Majewski S, Kaminska G, Bem W, Szmurlo A. Protease-mediated enhancement of lymphocyte-induced angiogenesis in X-ray irradiated mice. Int J Radiat Biol Relat Stud Phys Chem Med. 1983:43:149-56.

Kang JH, Kim EG, Kim W, Seong KM, Youn HS, Kim JW, Kim J, Youn BH. Rhamnetin and Cirsiliol Induce Radiosensitization and Inhibition of EpithelialMesenchymal Transition (EMT) by miR-34a-mediated Suppression of Notch-1 Expression in Non-small Cell Lung Cancer Cell Lines. Journal of Biological Chemistry. 2013;288(38):27343-57.

Kargiotis O, Chetty C, Gogineni V, Gondi CS, Pulukuri SM, Kyritsis AP, Gujrati M, Klopfenstein JD, Dinh DH, Rao JS. UPA/UPAR downregulation inhibits radiation-induced migration, invasion and angiogenesis in IOMM-lee meningioma cells and decreases tumor growth in vivo. Int J Oncol. 2008;33:937-47.

Kawamoto A, Yokoe T, Tanaka K, Saigusa S, Toiyama Y, Yasuda H, Inoue Y, Miki C, Kusunoki M. Radiation induces epithelial-mesenchymal transition in colorectal cancer cells. Oncol Rep. 2012;27:51-7.

Kim MY, Oskarsson T, Acharyya S, Nguyen DX, Zhang XH, Norton L, Massague J. Tumor self-seeding by circulating cancer cells. Cell. 2009;139:1315-26.

Kim RK, Cui YH, Yoo KC, Kim IG, Lee M, Choi YH, Suh Y, Lee SJ. Radiation promotes malignant phenotypes through SRC in breast cancer cells. Cancer Sci. 2015;106:78-85.

Kim R-K, Kaushik N, Suh Y, Yoo K-C, Cui Y-H, Kim M-J, Lee H-J, Kim I-G, Lee S-J. Radiation driven epithelial-mesenchymal transition is mediated by Notch signaling in breast cancer. Oncotarget. 2016;7(33).

Kioi M, Vogel H, Schultz G, Hoffman RM, Harsh GR, Brown JM. Inhibition of vasculogenesis, but not angiogenesis, prevents the recurrence of glioblastoma after irradiation in mice. J Clin Invest. 2010a;120:694-705.

Kioi M, Vogel H, Schultz G, Hoffman RM, Harsh GR, Martin Brown J. Inhibition of vasculogenesis, but not angiogenesis, prevents the recurrence of glioblastoma after irradiation in mice. Journal of Clinical Investigation. 2010b;120(3):694-705

Klein D, Schmetter A, Imsak R, Wirsdorfer F, Unger K, Jastrow H, Stuschke M, Jendrossek $V$. Therapy with multipotent mesenchymal stromal cells protects lungs from radiation-induced injury and reduces the risk of lung metastasis. Antioxid Redox Signal. 2016;24:53-69.
Koike A, Nakazato H, Moore GE. The fate of Ehrlich cells injected into the portal system. Surg Forum. 1962;13:113-5.

Kumar A, Collins H, Van Tam J, Scholefield JH, Watson SA. Effect of preoperative radiotherapy on matrilysin gene expression in rectal cancer. Eur J Cancer. 2002;38:505-10.

Kumar A, Collins HM, Scholefield JH, Watson SA. Increased type-IV collagenase (MMP-2 and MMP-9) activity following preoperative radiotherapy in rectal cancer. Br J Cancer. 2000;82:960-5.

Kuo YC, Wu HT, Hung JJ, Chou TY, Teng SC, Wu KJ. Nijmegen breakage syndrome protein 1 (NBS1) modulates hypoxia inducible factor-1alpha (HIF1alpha) stability and promotes in vitro migration and invasion under ionizing radiation. Int J Biochem Cell Biol. 2015a;64:229-38.

Kuo Y-C, Wu H-T, Hung J-J, Chou T-Y, Teng S-C, Wu K-J. Nijmegen breakage

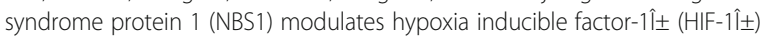
stability and promotes in vitro migration and invasion under ionizing radiation. The International Journal of Biochemistry \& Cell Biology. 2015b;64:229-38

Kuonen F, Laurent J, Secondini C, Lorusso G, Stehle J-C, Rausch T, Faes-van't Hull E, Bieler G, Alghisi G-C, Schwendener R, Andrejevic-Blant S, Mirimanoff R-O, Ruegg C. Inhibition of the Kit Ligand/c-Kit Axis Attenuates Metastasis in a Mouse Model Mimicking Local Breast Cancer Relapse after Radiotherapy. Clinical Cancer Research. 2012a;18(16):4365-74.

Kuonen F, Laurent J, Secondini C, Lorusso G, Stehle JC, Rausch T, Faes-Van't Hull E, Bieler G, Alghisi GC, Schwendener R, et al. Inhibition of the Kit ligand/c-Kit axis attenuates metastasis in a mouse model mimicking local breast cancer relapse after radiotherapy. Clin Cancer Res. 2012b;18:4365-74.

Kuonen F, Secondini C, Ruegg C. Molecular Pathways: Emerging Pathways Mediating Growth, Invasion, and Metastasis of Tumors Progressing in an Irradiated Microenvironment. Clinical Cancer Research. 2012c;18(19):5196-202.

Kuonen F, Secondini C, Ruegg C. Molecular pathways: emerging pathways mediating growth, invasion, and metastasis of tumors progressing in an irradiated microenvironment. Clin Cancer Res. 2012d;18:5196-202.

Lee HC, An S, Lee H, Woo SH, Jin HO, Seo SK, Choe TB, Yoo DH, Lee SJ, Hong YJ, et al. Activation of epidermal growth factor receptor and its downstream signaling pathway by nitric oxide in response to ionizing radiation. Mol Cancer Res. 2008:6:996-1002.

Lee $M$, Lee HJ, Seo WD, Park KH, Lee YS. Sialylation of integrin beta1 is involved in radiation-induced adhesion and migration in human colon cancer cells. Int J Radiat Oncol Biol Phys. 2010;76:1528-36.

Lee SY, Jeong EK, Ju MK, Jeon HM, Kim MY, Kim CH, Park HG, Han SI, Kang HS. Induction of metastasis, cancer stem cell phenotype, and oncogenic metabolism in cancer cells by ionizing radiation. Mol Cancer. 2017a;16:10

Lee SY, Jeong EK, Min Kyung J, Jeon HM, Kim MY, Kim CH, Park HG, Han SI, Kang HS. Induction of metastasis, cancer stem cell phenotype, and oncogenic metabolism in cancer cells by ionizing radiation. Molecular Cancer. 2017;16(1).

Lee SY, Jeong EK, Ju MK, Jeon HM, Kim MY, Kim CH, Park HG, Han SI, Kang HS. Induction of metastasis, cancer stem cell phenotype, and oncogenic metabolism in cancer cells by ionizing radiation. Molecular Cancer. 2017b;16(1).

Li D, Qu C, Ning Z, Wang H, Zang K, Zhuang L, Chen L, Wang P, Meng Z. Radiation promotes epithelial-to-mesenchymal transition and invasion of pancreatic cancer cell by activating carcinoma-associated fibroblasts. Am J Cancer Res. 2016a;6:2192-206.

Li D, Qu C, Ning Z, Wang H, Zang K, Zhuang L, Chen L, Wang P, Meng Z. Radiation promotes epithelial-tomesenchymal transition and invasion of pancreatic cancer cell by activating carcinoma-associated fibroblasts. Am J Cancer Res. 2016b;6(10):2192-206.

Li X, Ishihara S, Yasuda M, Nishioka T, Mizutani T, Ishikawa M, Kawabata K, Shirato $\mathrm{H}$, Haga $\mathrm{H}$. Lung cancer cells that survive ionizing radiation show increased integrin alpha2beta1- and EGFR-dependent invasiveness. PLoS One. 2013;8:e70905.

Liu J, Shen W, Tang Y, Zhou J, Li M, Zhu W, Yang H, Wu J, Zhang S, Cao J. Proteasome inhibitor MG132 enhances the antigrowth and antimetastasis effects of radiation in human nonsmall cell lung cancer cells. Tumour Biol. 2014a;35:7531-9.

Liu J, Shen W, Tang Y, Zhou J, Li M, Zhu W, Yang H, Wu J, Zhang S, Cao J. Proteasome inhibitor MG132 enhances the antigrowth and antimetastasis effects of radiation in human nonsmall cell lung cancer cells. Tumor Biology. 2014b;35(8):7531-9.

Liu W, Huang YJ, Liu C, Yang YY, Liu H, Cui JG, Cheng Y, Gao F, Cai JM, Li BL. Inhibition of TBK1 attenuates radiation-induced epithelial-mesenchymal 
transition of A549 human lung cancer cells via activation of GSK-3beta and repression of ZEB1. Lab Investig. 2014c;94:362-70.

Madani I, De Neve W, Mareel M. Does ionizing radiation stimulate cancer invasion and metastasis? Bull Cancer. 2008; 95(3):292-300.

Maier P, Hartmann L, Wenz F, Herskind C. Cellular pathways in response to ionizing radiation and their Targetability for tumor Radiosensitization. Int J Mol Sci. 2016;17:102.

Mareel M, Bracke M, Van Roy F, Vakaet L. Expression of E-cadherin in embryogenetic ingression and cancer invasion. Int J Dev Biol. 1993;37:227-35.

Mareel M, Oliveira MJ, Madani I. Cancer invasion and metastasis: interacting ecosystems. Virchows Arch. 2009a;454:599-622.

Mareel M, Oliveira MJ, Madani I. Cancer invasion and metastasis: interacting ecosystems. Virchows Archiv. 2009b;454(6):599-622.

Marley NF, Marley WM. Skin metastases in an area of radiation dermatitis. Arch Dermatol. 1982;118:129-31.

Meltzer J, Ahmed SA, Archambeau JO. The development of metastases within a field of previous irradiation: a case report. Cancer. 1981;48:717-20.

Merino OR, Lindberg RD, Fletcher GH. An analysis of distant metastases from squamous cell carcinoma of the upper respiratory and digestive tracts. Cancer. 1977:40:145-51.

Milas L, Hirata H, Hunter N, Peters LJ. Effect of radiation-induced injury of tumor bed stroma on metastatic spread of murine sarcomas and carcinomas. Cancer Res. 1988:48:2116-20.

Mole RH. Whole body irradiation; radiobiology or medicine? Br J Radiol. 1953;26:234-41.

Monnier Y, Farmer P, Bieler G, Imaizumi N, Sengstag T, Alghisi GC, Stehle JC, Ciarloni L, Andrejevic-Blant S, Moeckli R, et al. CYR61 and alphaVbeta5 integrin cooperate to promote invasion and metastasis of tumors growing in preirradiated stroma. Cancer Res. 2008;68:7323-31.

Murata K, Noda SE, Oike T, Takahashi A, Yoshida Y, Suzuki Y, Ohno T, Funayama T, Kobayashi Y, Takahashi T, Nakano T. Increase in cell motility by carbon ion irradiation via the rho signaling pathway and its inhibition by the ROCK inhibitor Y-27632 in lung adenocarcinoma A549 cells. J Radiat Res. 2014;55:658-64.

Nakayama A, Ninomiya I, Harada S, Tsukada T, Okamoto K, Nakanuma S, Sakai S, Makino I, Kinoshita J, Hayashi $H$, et al. Metformin inhibits the radiationinduced invasive phenotype of esophageal squamous cell carcinoma. Int J Oncol. 2016;49:1890-8.

Nalla AK, Asuthkar S, Bhoopathi P, Gujrati M, Dinh DH, Rao JS. Suppression of UPAR retards radiation-induced invasion and migration mediated by integrin beta1/FAK signaling in medulloblastoma. PLoS One. 2010:5:e13006.

Nielsen HM, Overgaard M, Grau C, Jensen AR, Overgaard J. Study of failure pattern among high-risk breast cancer patients with or without postmastectomy radiotherapy in addition to adjuvant systemic therapy: long-term results from the Danish breast Cancer cooperative group DBCG 82 b and c randomized studies. J Clin Oncol. 2006;24:2268-75.

Nozue M, Isaka N, Fukao K. Over-expression of vascular endothelial growth factor after preoperative radiation therapy for rectal cancer. Oncol Rep. 2001;8:1247-9.

Nubel T. Ionizing radiation-induced E-selectin gene expression and tumor cell adhesion is inhibited by lovastatin and all-trans retinoic acid. Carcinogenesis. 2004;25(8):1335-44.

Ogata T, Teshima T, Inaoka M, Minami K, Tsuchiya T, Isono M, Furusawa Y, Matsuura N. Carbon ion irradiation suppresses metastatic potential of human non-small cell lung Cancer A549 cells through the Phosphatidylinositol-3kinase/Akt signaling pathway. J Radiat Res. 2011;52:374-9.

Ogata T, Teshima T, Kagawa K, Hishikawa Y, Takahashi Y, Kawaguchi A, Suzumoto Y, Nojima K, Furusawa Y, Matsuura N. Particle irradiation suppresses metastatic potential of cancer cells. Cancer Res. 2005;65:113-20.

Ohuchida K, Mizumoto K, Murakami M, Qian LW, Sato N, Nagai E, Matsumoto K, Nakamura T, Tanaka M. Radiation to stromal fibroblasts increases invasiveness of pancreatic cancer cells through tumor-stromal interactions. Cancer Res. 2004;64:3215-22

O'Reilly MS, Holmgren L, Shing Y, Chen C, Rosenthal RA, Moses M, Lane WS, Cao Y, Sage EH, Folkman J. Angiostatin: a novel angiogenesis inhibitor that mediates the suppression of metastases by a Lewis lung carcinoma. Cell. 1994;79:315-28.

Paquette B, Therriault H, Desmarais G, Wagner R, Royer R, Bujold R. Radiationenhancement of MDA-MB-231 breast cancer cell invasion prevented by a cyclooxygenase-2 inhibitor. Br J Cancer. 2011;105:534-41.

Park CM, Park MJ, Kwak HJ, Lee HC, Kim MS, Lee SH, Park IC, Rhee CH, Hong SI. lonizing radiation enhances matrix metalloproteinase-2 secretion and invasion of glioma cells through Src/epidermal growth factor receptor-mediated p38/Akt and phosphatidylinositol 3-kinase/Akt signaling pathways. Cancer Res. 2006a;66:8511-9.
Park CM, Park MJ, Kwak HJ, Lee HC, Kim MS, Lee SH, Park IC, Rhee CH, Hong SI. lonizing radiation enhances matrix metalloproteinase-2 secretion and invasion of glioma cells through Src/epidermal growth factor receptormediated p38/Akt and phosphatidylinositol 3-kinase/Akt signaling pathways. Cancer Res. 2006b;66(17):8511-9.

Park JK, Jang SJ, Kang SW, Park S, Hwang SG, Kim WJ, Kang JH, Um HD. Establishment of animal model for the analysis of cancer cell metastasis during radiotherapy. Radiat Oncol. 2012;7:153.

Parthymou A, Kardamakis D, Pavlopoulos I, Papadimitriou E. Irradiated C6 glioma cells induce angiogenesis in vivo and activate endothelial cells in vitro. Int J Cancer. 2004;110:807-14.

Pickhard AC, Margraf J, Knopf A, Stark T, Piontek G, Beck C, Boulesteix AL, Scherer EQ, Pigorsch S, Schlegel J, et al. Inhibition of radiation induced migration of human head and neck squamous cell carcinoma cells by blocking of EGF receptor pathways. BMC Cancer. 2011a;11:388.

Pickhard AC, Margraf J, Knopf A, Stark T, Piontek G, Beck C, Boulesteix A-L, Scherer EQ, Pigorsch S, Schlegel J, Arnold W, Reiter R. Inhibition of radiation induced migration of human head and neck squamous cell carcinoma cells by blocking of EGF receptor pathways. BMC Cancer. 2011b;11(1)

Polytarchou C, Gligoris T, Kardamakis D, Kotsaki E, Papadimitriou E. X-rays affect the expression of genes involved in angiogenesis. Anticancer Res. 2004; $24: 2941-5$.

Poortmans PM, Collette S, Kirkove C, Van Limbergen E, Budach V, Struikmans H, Collette L, Fourquet A, Maingon P, Valli M, et al. Internal mammary and medial supraclavicular irradiation in breast Cancer. N Engl J Med. 2015;373:317-27.

Postow MA, Callahan MK, Barker CA, Yamada Y, Yuan J, Kitano S, Mu Z, Rasalan T, Adamow M, Ritter $\mathrm{E}$, et al. Immunologic correlates of the abscopal effect in a patient with melanoma. N Engl J Med. 2012;366:925-31.

Qian LW, Mizumoto K, Inadome N, Nagai E, Sato N, Matsumoto K, Nakamura T, Tanaka M. Radiation stimulates HGF receptor/c-met expression that leads to amplifying cellular response to HGF stimulation via upregulated receptor tyrosine phosphorylation and MAP kinase activity in pancreatic cancer cells. Int J Cancer. 2003;104:542-9.

Qian LW, Mizumoto K, Urashima T, Nagai E, Maehara N, Sato N, Nakajima M, Tanaka M. Radiation-induced increase in invasive potential of human pancreatic cancer cells and its blockade by a matrix metalloproteinase inhibitor, CGS27023. Clin Cancer Res. 2002;8:1223-7.

Rajput S, Kumar BN, Banik P, Parida S, Mandal M. Thymoquinone restores radiation-induced TGF-beta expression and abrogates EMT in chemoradiotherapy of breast cancer cells. J Cell Physiol. 2015;230:620-9.

Rajput S, Prashanth Kumar BN, Banik P, Parida S, Mandal M. Thymoquinone Restores Radiation-Induced TGF- $\left.\right|^{2}$ Expression and Abrogates EMT in Chemoradiotherapy of Breast Cancer Cells. Journal of Cellular Physiology. 2015;230(3):620-9.

Rieken S, Habermehl D, Mohr A, Wuerth L, Lindel K, Weber K, Debus J, Combs SE. Targeting alphanubeta3 and alphanubeta5 inhibits photon-induced hypermigration of malignant glioma cells. Radiat Oncol. 2011;6:132.

Rieken S, Habermehl D, Wuerth L, Brons S, Mohr A, Lindel K, Weber K, Haberer T, Debus J, Combs SE. Carbon ion irradiation inhibits glioma cell migration through downregulation of integrin expression. Int J Radiat Oncol Biol Phys. 2012:83:394-9.

Rofstad EK, Mathiesen B, Galappathi K. Increased metastatic dissemination in human melanoma xenografts after subcurative radiation treatment: radiation-induced increase in fraction of hypoxic cells and hypoxia-induced up-regulation of urokinase-type plasminogen activator receptor. Cancer Res. 2004;64:13-8.

Rofstad EK, Mathiesen B, Henriksen K, Kindem K, Galappathi K. The tumor bed effect: increased metastatic dissemination from hypoxia-induced up-regulation of metastasis-promoting gene products. Cancer Res. 2005;65:2387-96.

Russell JS, Brown JM. The irradiated tumor microenvironment: role of tumorassociated macrophages in vascular recovery. Front Physiol. 2013;4:157.

Russell JS, Martin Brown J. The irradiated tumor microenvironment: role of tumor-associated macrophages in vascular recovery. Frontiers in Physiology. 2013;4:1-9.

Schantz SP, Peters LJ. Patterns of recurrence from head and neck cancer. An immunologic perspective. Am J Clin Oncol. 1987;10:469-74.

Schweigerer L, Rave-Frank M, Schmidberger H, Hecht M. Sublethal irradiation promotes invasiveness of neuroblastoma cells. Biochem Biophys Res Commun. 2005;330:982-8.

Shaverdian N, Lisberg AE, Bornazyan K, Veruttipong D, Goldman JW, Formenti SC, Garon EB, Lee P. Previous radiotherapy and the clinical activity and toxicity of 
pembrolizumab in the treatment of non-small-cell lung cancer: a secondary analysis of the KEYNOTE-001 phase 1 trial. Lancet Oncol. 2017;18:895-903.

Sofia Vala I, Martins LR, Imaizumi N, Nunes RJ, Rino J, Kuonen F, Carvalho LM, Ruegg C, Grillo IM, Barata JT, et al. Low doses of ionizing radiation promote tumor growth and metastasis by enhancing angiogenesis. PLoS One. 2010;5:e11222.

Sonveaux P, Brouet A, Havaux X, Gregoire V, Dessy C, Balligand JL, Feron O. Irradiation-induced angiogenesis through the up-regulation of the nitric oxide pathway: implications for tumor radiotherapy. Cancer Res. 2003;63:1012-9.

Sosa MS, Bragado P, Aguirre-Ghiso JA. Mechanisms of disseminated cancer cell dormancy: an awakening field. Nat Rev Cancer. 2014;14:611-22.

Speake WJ, Dean RA, Kumar A, Morris TM, Scholefield JH, Watson SA. Radiation induced MMP expression from rectal cancer is short lived but contributes to in vitro invasion. Eur J Surg Oncol. 2005;31:869-74.

Stahler C, Roth J, Cordes N, Taucher-Scholz G, Mueller-Klieser W. Impact of carbon ion irradiation on epidermal growth factor receptor signaling and glioma cell migration in comparison to conventional photon irradiation. Int J Radiat Biol. 2013;89:454-61.

Stenstrom KW, Vermund H, Mosser DG, Marvin JF. Effects of roentgen irradiation on the tumor bed. I. The inhibiting action of local pretransplantation roentgen irradiation (1500 $\mathrm{r}$ alpha) on the growth of mouse mammary carcinoma. Radiat Res. 1955;2:180-91.

Storme GA, Mareel MM. Enhanced recovery from growth inhibition in irradiated M04 spheroids invading into embryonic chick heart fragments in organ culture. Cell Biol Int Rep. 1984;8:247-55.

Strong MS, Vaughan CW, Kayne HL, Aral IM, Ucmakli A, Feldman M, Healy GB. A randomized trial of preoperative radiotherapy in cancer of the oropharynx and hypopharynx. Am J Surg. 1978;136:494-500.

Su WH, Chuang PC, Huang EY, Yang KD. Radiation-induced increase in cell migration and metastatic potential of cervical cancer cells operates via the KRas pathway. Am J Pathol. 2012;180:862-71.

Sundahl N, De Wolf K, Rottey S, Decaestecker K, De Maeseneer D, Meireson A, Goetghebeur E, Fonteyne V, Verbeke S, De Visschere P, Reynders D, Van Gele M, Brochez L, Ost P. A phase I/II trial of fixed-dose stereotactic body radiotherapy with sequential or concurrent pembrolizumab in metastatic urothelial carcinoma: evaluation of safety and clinical and immunologic response. J Transl Med. 2017;15(1):150.

Sundahl N, De Wolf K, Kruse V, Meireson A, Reynders D, Goetghebeur E, Van Gele M, Speeckaert R, Hennart B, Brochez L, Ost P. Phase 1 Dose Escalation Trial of Ipilimumab and Stereotactic Body Radiation Therapy in Metastatic Melanoma. International Journal of Radiation Oncology*Biology*Physics. 2018;100(4):906-15.

Takahashi Y, Teshima T, Kawaguchi N, Hamada Y, Mori S, Madachi A, Ikeda S, Mizuno H, Ogata T, Nojima K, et al. Heavy ion irradiation inhibits in vitro angiogenesis even at sublethal dose. Cancer Res. 2003;63:4253-7.

Talmadge JE, Fidler IJ. AACR centennial series: the biology of cancer metastasis: historical perspective. Cancer Res. 2010;70:5649-69.

Tanaka Y. Effect of lung irradiation on the incidence of pulmonary metastases and its mechanism. Acta Radiol Ther Phys Biol. 1976;15:142-8.

Tang C, Welsh JW, de Groot P, Massarelli E, Chang JY, Hess KR, Basu S, Curran MA, Cabanillas ME, Subbiah V, et al. Ipilimumab with stereotactic ablative radiation therapy: phase I results and immunologic correlates from peripheral T cells. Clin Cancer Res. 2017;23:1388-96.

Timaner M, Bril R, Kaidar-Person O, Rachman-Tzemah C, Alishekevitz D, Kotsofruk R, Miller V, Nevelsky A, Daniel S, Raviv Z, et al. Dequalinium blocks macrophageinduced metastasis following local radiation. Oncotarget. 2015;6:27537-54.

Travis EL, Reinartz G, Chu AM, Down JD, Fowler JF. Effect of cyclophosphamide or $\mathrm{x}$-rays on spontaneously occurring metastases from tumors transplanted into the tails of mice. Cancer Res. 1981;41:1803-7.

Trudel D, Fradet Y, Meyer F, Tetu B. Matrix metalloproteinase 9 is associated with Gleason score in prostate cancer but not with prognosis. Hum Pathol. 2010;41:1694-701.

Tsujino K, Kashihara K, Kotani S, Hayakawa K, Imanaka K, Takada Y, Uno T, Hirata H, Kaneyasu Y, Sekiguchi K, et al. A survey of patients with inflammatory skin recurrence corresponding to the area of previous irradiation after postoperative radiotherapy for breast cancer. J Radiat Res. 2011;52:797-803.

Tsukamoto H, Shibata K, Kajiyama H, Terauchi M, Nawa A, Kikkawa F. Irradiationinduced epithelial-mesenchymal transition (EMT) related to invasive potential in endometrial carcinoma cells. Gynecol Oncol. 2007;107:500-4.

Tsutsumi K, Tsuda M, Yazawa N, Nakamura H, Ishihara S, Haga H, Yasuda M, Yamazaki R, Shirato $H$, Kawaguchi $H$, et al. Increased motility and invasiveness in tumor cells that survive 10 Gy irradiation. Cell Struct Funct. 2009;34:89-96.

Twyman-Saint Victor C, Rech AJ, Maity A, Rengan R, Pauken KE, Stelekati E, Benci JL, Xu B, Dada H, Odorizzi PM, et al. Radiation and dual checkpoint blockade activate non-redundant immune mechanisms in cancer. Nature. 2015;520:373-7.
Vacchelli E, Bloy N, Aranda F, Buque A, Cremer I, Demaria S, Eggermont A, Formenti SC, Fridman WH, Fucikova J, et al. Trial watch: immunotherapy plus radiation therapy for oncological indications. Oncoimmunology. 2016;5:e1214790.

Vakaet LAM-L, Boterberg T. Pain control by ionizing radiation of bone metastasis. The International Journal of Developmental Biology. 2004;48(5-6):599-606.

Vehlow A, Cordes N. Invasion as target for therapy of glioblastoma multiforme. Biochim Biophys Acta. 1836;2013:236-44.

Vilalta M, Rafat M, Giaccia AJ, Graves EE. Recruitment of circulating breast cancer cells is stimulated by radiotherapy. Cell Rep. 2014;8:402-9.

Vilalta M, Rafat M, Graves EE. Effects of radiation on metastasis and tumor cell migration. Cellular and Molecular Life Sciences. 2016;73(16):2999-3007.

Wang SC, Yu CF, Hong JH, Tsai CS, Chiang CS. Radiation therapy-induced tumor invasiveness is associated with SDF-1-regulated macrophage mobilization and vasculogenesis. PLoS One. 2013;8:e69182.

Wick W, Wick A, Schulz JB, Dichgans J, Rodemann HP, Weller M. Prevention of irradiation-induced glioma cell invasion by temozolomide involves caspase 3 activity and cleavage of focal adhesion kinase. Cancer Res. 2002;62:1915-9.

Wild-Bode C, Weller M, Rimner A, Dichgans J, Wick W. Sublethal irradiation promotes migration and invasiveness of glioma cells: implications for radiotherapy of human glioblastoma. Cancer Res. 2001;61:2744-50.

Willaert W, Mareel M, Van De Putte D, Van Nieuwenhove Y, Pattyn P, Ceelen W. Lymphatic spread, nodal count and the extent of lymphadenectomy in cancer of the colon. Cancer Treatment Reviews. 2014;40(3):405-13.

Williams NL, Wuthrick EJ, Kim H, Palmer JD, Garg S, Eldredge-Hindy H, Daskalakis C, Feeney KJ, Mastrangelo MJ, Kim LJ, et al. Phase 1 study of Ipilimumab combined with whole brain radiation therapy or radiosurgery for melanoma patients with brain metastases. Int J Radiat Oncol Biol Phys. 2017;99:22-30.

Withers HR, Milas L. Influence of preirradiation of lung on development of artificial pulmonary metastases of fibrosarcoma in mice. Cancer Res. 1973;33:1931-6.

Yan S, Wang Y, Yang Q, Li X, Kong X, Zhang N, Yuan C, Yang N, Kong B. Low-dose radiation-induced epithelial-mesenchymal transition through NF-kappaB in cervical cancer cells. Int J Oncol. 2013a;42:1801-6.

Yan S, Wang Y, Yang Q, Li X, Kong X, Zhang N, Yuan C, Yang N, Kong B. Low-dose radiation-induced epithelial-mesenchymal transition through NF-1̂oB in cervical cancer cells. International Journal of Oncology. 2013b; 42(5):1801-6.

Yao H, Zeng ZZ, Fay KS, Veine DM, Staszewski ED, Morgan M, Wilder-Romans K, Williams TM, Spalding AC, Ben-Josef E, Livant DL. Role of alpha(5)beta(1) integrin up-regulation in radiation-induced invasion by human pancreatic Cancer cells. Transl Oncol. 2011;4:282-92.

Yuan W, Yuan Y, Zhang T, Wu S. Role of Bmi-1 in regulation of ionizing irradiation-induced epithelial-mesenchymal transition and migration of breast cancer cells. PLoS One. 2015a;10:e0118799.

Yuan W, Yuan Y, Zhang T, Wu S, Zhang Z. Role of Bmi-1 in Regulation of lonizing Irradiation-Induced Epithelial-Mesenchymal Transition and Migration of Breast Cancer Cells. PLOS ONE. 2015b;10(3):e0118799.

Zhai GG, Malhotra R, Delaney M, Latham D, Nestler U, Zhang M, Mukherjee N, Song Q, Robe P, Chakravarti A. Radiation enhances the invasive potential of primary glioblastoma cells via activation of the rho signaling pathway. J Neuro-Oncol. 2006a;76:227-37.

Zhai GG, Malhotra R, Delaney M, Latham D, Nestler U, Zhang M, Mukherjee N, Song Q, Robe P, Chakravarti A. Radiation Enhances the Invasive Potential of Primary Glioblastoma Cells via Activation of the Rho Signaling Pathway. Journal of Neuro-Oncology. 2006b;76(3):227-37.

Zhang AX, Lu FQ, Yang YP, Ren XY, Li ZF, Zhang W. MicroRNA-217 overexpression induces drug resistance and invasion of breast cancer cells by targeting PTEN signaling. Cell Biol Int. 2015.

Zhang X, Li X, Zhang N, Yang Q, Moran MS. Low doses ionizing radiation enhances the invasiveness of breast cancer cells by inducing epithelialmesenchymal transition. Biochem Biophys Res Commun. 2011;412:188-92.

Zhang X, Zheng L, Sun Y, Wang T, Wang B. Tangeretin enhances radiosensitivity and inhibits the radiation-induced epithelial-mesenchymal transition of gastric cancer cells. Oncol Rep. 2015;34:302-10.

Zhao Q, Mao A, Guo R, Zhang L, Yan J, Sun C, Tang J, Ye Y, Zhang Y, Zhang H. Suppression of radiation-induced migration of non-small cell lung cancer through inhibition of Nrf2-notch Axis. Oncotarget. 2017:8:36603-13.

Zheng Q, Liu Y, Zhou HJ, Du YT ZBP, Zhang J, Miao GY, Liu B, Zhang H. X-ray radiation promotes the metastatic potential of tongue squamous cell carcinoma cells via modulation of biomechanical and cytoskeletal properties. Hum Exp Toxicol. 2015;34:894-903. 
Zhou LY, Wang ZM, Gao YB, Wang LY, Zeng ZC. Stimulation of hepatoma cell invasiveness and metastatic potential by proteins secreted from irradiated nonparenchymal cells. Int J Radiat Oncol Biol Phys. 2012;84:822-8.

Zhou YC, Liu JY, Li J, Zhang J, Xu YQ, Zhang HW, Qiu LB, Ding GR, Su XM, Mei S, Guo GZ. lonizing radiation promotes migration and invasion of cancer cells through transforming growth factor-beta-mediated epithelial-mesenchymal transition. Int J Radiat Oncol Biol Phys. 2011a;81:1530-7.

Zhou YC, Liu JY, Li J, Zhang J, Xu YQ, Zhang HW, Qiu LB, Ding GR, Su XM, Shi M, Guo GZ. Ionizing radiation promotes migration and invasion of cancer cells through transforming growth factor-beta-mediated epithelialmesenchymal transition. Int. J Radiat Oncol Biol Phys. 2011 b;81(5):1530-7.

Submit your next manuscript to BioMed Central and we will help you at every step:

- We accept pre-submission inquiries

- Our selector tool helps you to find the most relevant journal

- We provide round the clock customer support

- Convenient online submission

- Thorough peer review

- Inclusion in PubMed and all major indexing services

- Maximum visibility for your research

Submit your manuscript at www.biomedcentral.com/submit
Biomed Central 